\title{
On the clock of the combinatorial clock auction
}

\author{
MAARTEN JANSSEN \\ Department of Economics, University of Vienna, National Research University Higher School of \\ Economics Moscow, and CEPR \\ Bernhard Kasberger \\ The Queen's College, University of Oxford
}

The combinatorial clock auction (CCA) has frequently been used in recent spectrum auctions. It combines a dynamic clock phase and a one-off supplementary round. The winning allocation and the corresponding prices are determined by the Vickrey-Clarke-Groves rules. These rules should encourage truthful bidding, whereas the clock phase is intended to reveal information. We inquire into the role of the clock when bidders have lexicographic preferences for raising rivals' costs. We show that in an efficient equilibrium, the clock cannot fully reveal bidders' types. In the spirit of the ratchet effect, in the supplementary round competitors extract surplus from strong bidders whose type is revealed. We also show that if there is substantial room for information revelation, that is, if the uncertainty about the final allocation is large, all equilibria of the CCA are inefficient. Qualitative features of our equilibria are in line with evidence concerning bidding behavior in some recent CCAs.

KeYwords. Combinatorial auctions, spectrum auctions, spiteful bidding, raising rival's cost, ratchet effect.

JEL CLASSification. D44, D47, L96.

\section{Introduction}

In recent years, many regulators around the world have chosen the combinatorial clock auction (CCA) to allocate telecommunication spectrum. The CCA has partially replaced the older simultaneous ascending auction (SAA) for two reasons. First, in the SAA, bidders may strategically reduce demand. If it is relatively clear to the bidders what the final allocation of an auction is where bidders bid competitively, then they have an incentive to reach the same allocation at much lower prices (Grimm et al. 2003). The SAA

\footnotetext{
Maarten Janssen: maarten.janssen@univie.ac . at

Bernhard Kasberger: bernhard. kasberger@queens.ox.ac .uk

Earlier versions of this paper were presented in Vienna (Workshop on Auction Design, 2016), Cardiff (QED Jamboree, 2015), Klagenfurt (NOeG, 2015), Istanbul (Conference on Economic Design, 2015), Montreal (11th World Congress of the Econometric Society, 2015), Munich (EARIE, 2015), and Cologne (Workshop on Auctions and Procurement Design, 2015). We thank audiences at these workshops and conferences, the two anonymous referees, Larry Ausubel, Oleg Baranov, Martin Bichler, Peter Cramton, Jon Levin, Paul Milgrom (and two of his graduate students), and Andy Skrzypacz for useful suggestions and comments. This research was supported by the Oesterreichische Nationalbank (Oesterreichische Nationalbank, Anniversary Fund, project number 15994).

(C) 2019 The Authors. Licensed under the Creative Commons Attribution-NonCommercial License 4.0. Available at http://econtheory.org. https://doi.org/10.3982/TE3203
} 
provides bidders with the possibility of reaching such a noncompetitive outcome. The sophisticated design of the CCA should overcome this issue as it incorporates (i) a generalized second-price (Vickrey) rule providing bidders with an incentive to bid truthfully (Cramton 2013) and (ii) a clock phase that should facilitate "price and package discovery" (Ausubel et al. 2006). Second, in contrast to the SAA, bidders can express bids for packages in the CCA. Package bidding is deemed to be important as current spectrum auctions allocate multiple units where bidders may value a package of licenses more than the sum of the individual components. If that is the case, the SAA, but not the CCA, suffers from the well known exposure problem, i.e., at the end of the auction, bidders may end up with a few units at a price that is more than their value for these units. The focus of this paper is the first issue: is it the case that the CCA provides bidders with an incentive to bid truthfully and that the clock phase facilitates price and package discovery?

The CCA is a dynamic version of the Vickrey-Clarke-Groves (VCG) mechanism and consists of two integrated phases. ${ }^{1}$ In the first clock phase, bidders express their demand on packages at given prices in every round. If, for a certain good, demand is larger than supply in a given round, then the price for that good is increased in the next round. The clock phase ends when demand is not larger than supply for all the auctioned goods. Importantly, no goods are allocated and no prices are determined at the end of the clock phase. Instead, the clock phase imposes constraints on the bids that are allowed in the second, supplementary, phase. In that one-off sealed-bid phase, bidders can bid on as many additional packages as they like and they may raise bids on packages they have bid on in the clock phase. At the end of the supplementary phase, goods are allocated and prices are determined. The auctioneer uses all the bids from the clock phase and the supplementary phase to determine the value-maximizing combination of bids, while the Vickrey pricing rule determines the prices winners pay.

Without the clock phase, the CCA reduces to the VCG mechanism. As the number of packages is an exponential function of the number of commodities, bidders in a VCG auction may need to consider bidding on a vast number of packages. In particular, if the uncertainty concerning competitors is large, bidders may have a fairly limited idea about the package they may eventually win and at which price. Through "price and package discovery," the clock phase is meant to reveal this kind of information. Bidders can then focus their bidding in the supplementary round on the packages that may still be winning.

Under standard preferences, truthful bidding in the clock and supplementary phase is indeed an equilibrium. If bidders bid truthfully, the outcome is efficient. However, truthful bidding is not a strict equilibrium, as bidders may be indifferent across many permissible bids in the supplementary round (Levin and Skrzypacz 2016). To eliminate the payment relevant indifferences, we consider bidders who ceteris paribus prefer outcomes where competitors pay more. We model this objective as a secondary dimension in a lexicographic way.

\footnotetext{
${ }^{1}$ In practice, there is a third phase: the assignment phase. In this phase, generic packages are allocated. We abstract away from this phase since it does not affect our analysis.
} 
Our first main result is that if bidders have a lexicographic preference for raising rival's costs, an efficient fully revealing equilibrium does not exist. This result implies that the CCA exhibits a fundamental trade-off between efficiency and information revelation in the clock phase. The trade-off follows from the fact that if bidders bid truthfully in the clock phase, the clock fully reveals information about the bidders' types. Bidders would like to use this information to maximally raise the rival's cost by placing bids in the supplementary phase on large packages that they know cannot be winning. The stronger their competitor, the more they can raise their price. The rules of the CCA are such that bidders are only able to raise the rival's cost if they expand demand in the clock phase, as this relaxes the constraints on the supplementary phase bids. Predicting that the clock phase eventually will fully reveal information, bidders can expand demand in the early phase of the clock without the risk of affecting the final allocation. Knowing that the competitor is able and inclined to raise their cost if their types are fully revealed, stronger bidders have an incentive to pool with weaker types in the clock phase.

This result is best understood from the perspective of the ratchet effect known from the dynamic principal-agent literature (Laffont and Tirole 1988). In that literature, an agent may have an incentive not to reveal his type to a principal if the principal could use that information to extract more surplus from the agent in future interactions. In our case, knowing the competitor is strong, a bidder (by bidding more aggressively in the supplementary phase) may increase the price the competitor has to pay beyond what it would be if the competitor's type were unknown. Rationally anticipating this exploitation, stronger bidders prefer to pool with weaker types. The intuition for our first main result differs in two dimensions from the traditional ratchet effect. First, unlike the principal-agent model, the roles of bidders in an auction are symmetric to one another so that each bidder is both the object of and the initiator of surplus extraction. Second, the extent to which bidders can raise the rival's cost in the supplementary round is not exogenously given, but endogenously determined by their behavior in the clock phase. Thus, bidders will only be able to raise the rival's cost if they expand demand in the clock phase.

The result that fully revealing efficient equilibria do not exist does not rule out the existence of efficient equilibria. Even with lexicographic preferences for raising rival's cost, efficient equilibria exist. We present examples of efficient equilibria, where to be able to raise rival's cost, bidders demand the full supply (even if prices are such that truthful bidding would tell them to demand less). ${ }^{2}$ The demand expansion phase ends with a sudden switch to truthful bidding. In one type of equilibrium, the clock stops immediately when all bidders drop demand. In such an equilibrium, there is no price or package discovery whatsoever. This clock phase development allows all bidders to bid their true marginal values in the supplementary round. As a result, the final allocation is efficient. We show that any efficient equilibrium of the CCA involves this type of demand expansion in the clock phase.

\footnotetext{
${ }^{2}$ This is in line with, for example, the Austrian 2013 auction, where (as we mention below) bidders were bidding very aggressively in the clock phase.
} 
Our second main result is that if the uncertainty concerning the competitor's type is sufficiently large, all equilibria of the CCA are inefficient. Efficiency and the high uncertainty require that weak bidders drop out at relatively high prices. Due to the spite motive, some strong bidders expand demand prior to these dropout prices. When the clock does not end, a relatively strong bidder infers from the continuation of the clock that the competitor is not too weak. This type of learning creates the opportunity for the strong bidder to make the supplementary round behavior conditional on the price at which the clock phase stops. Knowing the competitor is not too weak, the strong bidder can raise the rival's cost more without running the risk of winning an inferior share. Consequently, some types have an incentive to obfuscate their type and do this by reducing demand toward the end of the clock phase. This demand reduction rules out expressing true marginal values for all shares in the supplementary round, resulting in an inefficient final allocation. As we also show that the static VCG mechanism always has efficient equilibria, we claim that it is the clock phase that creates this inefficiency. ${ }^{3}$

Ausubel (2004, p. 1452) states that "the auctions literature has provided us with two fundamental prescriptions guiding effective auction design": first, "the winner's price should depend solely on opposing participants' bids-as in the sealed-bid, second-price auction-so that each participant has full incentive to reveal truthfully her value for the good. Second, an auction should be structured in an open fashion that maximizes the information made available to each participant at the time she places her bids." Our results show that following these two prescriptions can be at the expense of generating efficient outcomes in multi-unit auctions where bidders have a weak incentive to raise rival's costs. If efficiency is preserved, then the information that is revealed through the open format is fairly limited.

The lexicographic modeling of bidders' preference for raising the rival's costs implies that if two bidding strategies yield the same expected surplus to a bidder, the bidder chooses the strategy where the rival pays more. ${ }^{4}$ The motivation to raise rival's costs may arise from (i) principal-agent issues within a firm (bidder) ${ }^{5}$ or from (ii) the fact that (in spectrum auctions) bidders face weaker competitors in the market after an auction if competitors have paid more for their licenses. If firm A makes firm B pay more for spectrum, B's credit rating may fall and its cost of capital may go up, weakening its strategic position. Milgrom (2004) and Cramton and Ockenfels (2017) mention fairness as a reason why bidders may want to raise rival's costs.

\footnotetext{
${ }^{3}$ Note that as we do not present an alternative auction model that is clearly better than the CCA (or the SAA), the CCA cannot be fully discarded on these grounds. Nevertheless, it is important to understand that the CCA rules can be gamed and this may have consequences.

${ }^{4}$ The analysis with lexicographic preferences provides a robustness check on the equilibria under standard preferences: equilibria under our preferences are also equilibria under standard preferences, but the reverse does not necessarily hold true.

${ }^{5}$ In spectrum auctions, given the considerable uncertainty concerning future technological developments and uptake of data services, it is difficult for bidders to evaluate what the spectrum is worth. Valuations are highly subjective. Accordingly, if a bidder wants to have a more objective evaluation measure of his bidding team's performance, it might be better to evaluate performance relative to other bidders than relative to his own uncertain and subjective valuation.
} 
The motivation to raise rival's costs motive has become a concern in designing auctions. ${ }^{6}$ After the 2013 auction, the Austrian regulator RTR attributed the high revenue to overly aggressive behavior by bidders: during the clock phase, bidders were bidding very aggressively, and the majority of the supplementary bids were on very large packages that had a low probability of winning but played a crucial role in determining other bidders' prices. The fact that payments in the Austrian auction were essentially the same as the final clock prices is a clear signal of aggressive bidding, as with Vickrey pricing and "downward sloping demand" one would not expect marginal and average prices to be identical. The observed behavior, however, is reminiscent of the equilibria we describe. Moreover, the British regulator Ofcom (2014, p. 38, 6.73-6.77) explicitly mentions the possibility of price driving by placing "risk-free bids" in the supplementary phase as a problematic aspect of the CCA. Some of the potential bidders' responses share this concern (e.g., BT 2015). None of these arguments for raising rival's costs implies that bidders should have a lexicographic preference for doing so; lexicographic preferences are, however, a useful modeling approach to inquire into the robustness of the results of the CCA to slight changes in assumptions regarding preferences.

This is the first paper that provides a full equilibrium analysis of the CCA when bidders have a lexicographic preference for raising rival's costs. The most closely related paper is Levin and Skrzypacz (2016). They put forward a sequence of three related models in which, as in our study, two players compete for a perfectly divisible good in the CCA. In some parts of their analysis, they also consider spiteful bidders.

In a first model where bidders have standard preferences, Levin and Skrzypacz (2016) elegantly uncover the existence of multiple equilibria due to a key indifference condition. Both bidders use linear proxy clock demand functions so that the clock ends with market clearing. The activity rules then permit a specific range of supplementary bids that are all such that the final clock allocation is the final allocation. As the activity rules fix the allocation, the VCG pricing scheme makes bidders with standard preferences indifferent across all supplementary bids. How bidders resolve the indifference impacts optimal clock behavior, leading to a multiplicity of equilibria.

This indifference partly motivates Levin and Skrzypacz (2016) to consider spiteful lexicographic preferences in their next two models. In their second model, they (exogenously) restrict one bidder to linear proxy strategies. It is, however, not clear why one of the ex ante symmetric bidders would prefer to restrict himself and take this disadvantageous role. In their online Appendix, Levin and Skrzypacz (2016) discuss a third model with two predatory bidders. This model is closest to the model we analyze in our paper. In that third model, Levin and Skrzypacz (2016) have bidders using linear proxy strategies in the clock phase, but weaken the constraints on supplementary bids implied by this clock behavior and the activity rules. Technically, they achieve this by introducing an exogenous parameter that measures how much bidders violate the activity rules. Importantly, such bidding behavior violates the rules of the CCA (see Figure 1 for more detail).

\footnotetext{
${ }^{6}$ See, e.g., (i) Levin and Skrzypacz (2016) on the outcome of the Swiss auction and the discussion on why Sunrise paid much more for comparable spectrum than other bidders, and (ii) Ofcom (2012, p. 122, point 7.9) in response to an earlier consultation on the U.K. spectrum auction in 2013.
} 
In contrast, the focus of our paper is on how bidders behave in the clock phase so that they are able, within the rules of the CCA, to weaken the constraints of the activity rule and submit spiteful supplementary bids. We show that this is not innocuous, as our results are qualitatively and quantitatively different from the findings of Levin and Skrzypacz (2016). First, where Levin and Skrzypacz (2016) conclude that equilibria are inefficient, we show that efficient equilibria can exist if the uncertainty concerning bidders' types is not too large. In any efficient equilibrium, bidders endogenously relax the constraints of the activity rule through demand expansion in the clock phase. In this way, the clock does not perfectly reveal bidders' types and may end with excess supply so that the last clock round does not fix the final allocation. In the supplementary phase, bidders then have a strong incentive to bid true marginal values on possible final shares. Second, where we observe inefficient equilibria for large uncertainty, the source of inefficiency is very different from that in Levin and Skrzypacz (2016), where the source of inefficiency is the best response to exogenously distorted marginal prices; the inefficiency in our model derives from the incentives of strong bidders to obfuscate their types (as in the literature on the ratchet effect in the dynamic principal-agent literature) to avoid being exploited in the supplementary round.

While we consider the interaction between the clock and the supplementary phase, Janssen and Karamychev (2016) focus only on the supplementary phase of the CCA. Assuming a particular clock phase behavior, they show how the supplementary phase can be solved using iterative elimination of dominated strategies, resulting in bidders raising rival's costs without running the risk of winning undesired packages. The current paper analyzes the equilibrium properties of both stages of the CCA, i.e., the entire game.

A variant of the CCA was first suggested by Ausubel et al. (2006) and further developed in Cramton (2013). Ausubel and Baranov (2014) discuss the evolution of the CCA. Gretschko et al. (2017) discuss why bidding can be complicated in a CCA. Bichler et al. (2013) report experimental evidence on the CCA and present a simple example in which one bidder submits a spiteful bid.

The rest of this paper is organized as follows. Section 2 describes the auction rules and the model. Section 3 proves our first main result that there do not exist efficient equilibria of the CCA where the clock phase fully reveals bidders' types. Section 4 presents our second main result, namely that if the uncertainty concerning the competitor's type is large, the CCA does not have efficient equilibria. Both sections present general propositions and illustrate the main results through examples of equilibria. The examples also show that the nonexistence of equilibria that satisfy certain properties is not due to a general nonexistence of equilibria. Section 5 analyzes the VCG mechanism as a benchmark. We show that under standard preferences, iterated elimination of weakly dominated strategies always results in an efficient outcome, but it leaves the bids of weak types on large shares undetermined. Lexicographic preferences impose that these bids are chosen to raise rival's costs. Section 6 concludes with a discussion where we also consider the relevance of our paper for interpreting real-world auctions. Most proofs are provided in the Appendix. 


\section{Auction Rules AND THE MOdel}

We consider auctions where two bidders compete to get a share $x_{i} \in[0,1]$ of a unit of a divisible good. Throughout the paper, when a bidder has label $i=1,2$, the other bidder's label is $j=3-i$. As the VCG auction is an important part of the CCA, we first describe the rules of the VCG auction before we go into the details of the CCA. After presenting the auction rules, we describe our assumptions regarding each bidder's preferences.

VCG rules In the VCG auction, all bidders simultaneously submit their bids for all shares, that is, bidder $i$ submits a bidding function $S_{i}:[0,1] \rightarrow \mathbb{R}_{+}$. Bidders cannot bid a positive amount on getting nothing, i.e., $S_{i}(0)=0$. Subsequently, the auctioneer chooses the allocation $\mathbf{x}=\left(x_{1}, x_{2}\right)$ that maximizes the sum of bids, i.e., $\mathbf{x} \in \arg \max _{x} S_{1}\left(x_{1}\right)+$ $S_{2}\left(x_{2}\right)$ such that $x_{1}+x_{2} \leq 1$ and $x_{i} \geq 0$ for $i=1,2$. If two or more allocations solve the maximization problem, the auctioneer implements the allocation that minimizes the distance to the allocation $(1 / 2,1 / 2)$.

Bidder $i$ receives share $x_{i}$ and pays the VCG price $\max _{y} S_{j}(y)-S_{j}\left(x_{j}\right)$, i.e., the opportunity cost he (reportedly) imposes on the other bidder. When there is no possibility of confusion, we sometimes drop subscripts. Hence, with strictly increasing bidding functions, the final allocation is $(x, 1-x)$ and bidder $i$ has to pay $S_{j}(1)-S_{j}(1-x)$. Throughout the paper, we use the bid on the full supply to raise the rival's costs.

CCA rules The CCA is an auction with two stages. In the first stage, the clock phase, the auctioneer successively increases the price of the good and bidders report demands. The second, supplementary stage is a VCG auction where, in addition to the rules specified above, the bids are subject to so-called activity rules that are described below. Put differently, the clock phase elicits a demand function, whereas in the supplementary phase, bidders submit an inverse demand function. Activity rules aim for the consistency of the two functions. As explained in the Introduction, the rationale of the clock phase is price and package discovery, while VCG pricing should incentivize truthful bidding (Cramton 2013). The supplementary phase is meant to avoid some units remaining unsold and to allow bidders to express their preferences better.

At each point of time in the clock phase, the auctioneer announces a price and bidders report the share they demand at current prices. If aggregate demand is larger than supply, the price is increased. The clock ends as soon as there is no excess demand so that the clock can end with market clearing or excess supply. Importantly, bidders are not allowed to increase their demand during the clock phase. We model this in the following way. The clock phase begins at an initial price $p_{0}=0$. The clock price is increased continuously as long as there is excess demand. Bidder $i$ 's action in the clock phase is a weakly decreasing demand function $x_{i}: \mathbb{R}_{+} \rightarrow[0,1]$ that maps prices to demand. The clock phase stops at $\tilde{p}$ if excess demand is smaller than or equal to zero, i.e., if $x_{1}(\tilde{p})+x_{2}(\tilde{p}) \leq 1$.

In the main part of the paper, we analyze a CCA where bidders do not receive any information concerning aggregate demand in the clock phase. ${ }^{7}$ Hence, each bidder can

\footnotetext{
${ }^{7}$ Real-world CCAs have used different regimes concerning information disclosure in the clock phase. In one regime, bidders are only informed about the fact that there is still excess demand and that the clock
} 
only condition his demand on the price, but not on his rival's previous demand. This assumption facilitates the formal analysis of the auction. We also comment, however, on an information policy where the last clock round demands are announced. At the end of Section 4, we present an example of an inefficient equilibrium under this information policy.

In the supplementary phase, bidders can submit bids on all possible shares, that is, they submit bidding functions $S_{i}:[0,1] \rightarrow \mathbb{R}_{+}$. Given the supplementary bids, the auctioneer uses the same rules as described above for the VCG mechanism to compute the final allocation and individual CCA prices. ${ }^{8}$

Importantly, the CCA has activity rules linking the clock and the supplementary phase by translating the clock demand behavior into constraints on the supplemental bids. More specifically, the supplementary bidding function $S_{i}$ must satisfy three types of constraints. First, clock bids remain valid, i.e., if bidder $i$ demanded $x$ at clock price $p$, then it has to be the case that $S_{i}(x) \geq p \cdot x$. Unlike for all other bids, there are no further constraints for the bid on the final clock round demand. Second, the so-called final cap rule requires that supplementary bids satisfy the axiom of revealed preference with respect to the final clock round demand, i.e., $S_{i}(x) \leq S_{i}\left(\tilde{x}_{i}\right)+\tilde{p}\left(x-\tilde{x}_{i}\right), x \neq \tilde{x}_{i}$, where $\tilde{p}$ is the final clock round price and $\tilde{x}_{i}$ is bidder $i$ 's demand in the final clock round. Finally, the relative cap requires that if in the clock phase bidder $i$ demanded $x$ at a price $p$, then for any $x^{\prime}>x$, bidder $i$ cannot express an incremental bid for $x^{\prime}$ in the supplementary round that is larger than $p$, i.e., $S_{i}\left(x^{\prime}\right) \leq S_{i}(x)+p\left(x^{\prime}-x\right)$. A bid on $x^{\prime}$ cannot be larger than the area under the expressed clock demand curve. ${ }^{9}$

The intuitive rationale behind these three activity rules is as follows. The first rule requiring that clock bids remain valid is a minimal requirement to make clock bids meaningful. The final cap rule guarantees that if the clock ends with market clearing, the final clock allocation is the final allocation. As bidders do not know in advance when is the last clock round, this rule encourages bidders to bid truthfully in the clock. ${ }^{10}$ Finally, the relative cap rule motivates bidders when choosing between two different packages to bid according to their relative preference evaluated at the current round prices. Because of the second price rule, it is considered that bidders have an incentive to bid value on all possible packages in the supplementary round. The final cap and relative cap are such that by bidding truthfully in the clock, bidders can bid value in the supplementary phase.

phase continues. In another regime, bidders are informed about aggregate demand in every clock round. The first regime was used in the first part of the Austrian auction and seems to be favored if collusion between bidders might be an issue. In the consultation document on the award of the $2.3 \mathrm{GHz}$ and $3.4 \mathrm{GHz}$ bands, Ofcom (2014) proposed using either the CCA or the SAA without demand disclosure. In a reaction for Hutchinson 3G, Power Auctions LLC (2015) claims that a dynamic auction with no demand disclosure is basically a sealed-bid auction. We show, however, that even without demand disclosure, the equilibrium outcomes that can be sustained in a CCA differ from the outcomes of the VCG.

${ }^{8}$ We do not consider the "core-selecting" elements in the pricing rule of real-world CCAs (see, e.g., Day and Milgrom 2008, Day and Cramton 2012, and Erdil and Klemperer 2010, as well as Goeree and Lien 2016 and Ausubel and Baranov 2019).

${ }^{9}$ Levin and Skrzypacz (2016) provide a figure that illustrates the activity rules.

${ }^{10}$ Below, we formally define what it means to bid truthfully in the clock. 


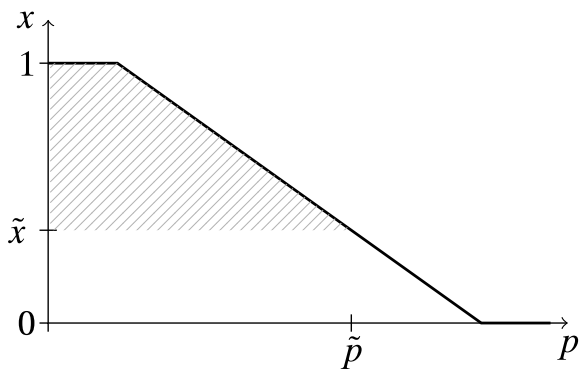

(a) Truthful Bidding

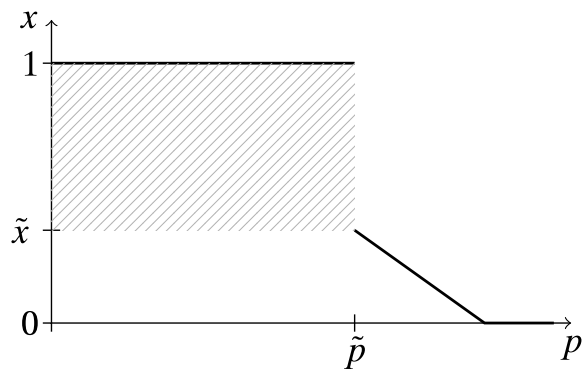

(b) Demand Expansion

FIGURE 1. Clock demand and constraints of the activity rules.

Preferences and information The utility a bidder derives from acquiring a share $x$ is denoted by $U\left(x, \theta_{i}\right)$, where $\theta_{i}$ is bidder $i$ 's privately known type. A bidder's type is randomly drawn from an atomless and commonly known distribution with support $[\underline{\theta}, \bar{\theta}]$. The set of type profiles $\theta=\left(\theta_{1}, \theta_{2}\right)$ is denoted by $[\underline{\theta}, \bar{\theta}]^{2}$. The utility function $U\left(x, \theta_{i}\right)$ is strictly increasing in $\theta_{i}$ and $x$, twice continuously differentiable, and concave in $x$. The marginal utility is increasing in $\theta_{i}$, i.e., $\partial^{2} U\left(x, \theta_{i}\right) / \partial \theta_{i} \partial x>0$ for $x>0$ and nonnegative for $x=0$. When convenient, we write $U_{i}(x)$ instead of $U\left(x, \theta_{i}\right)$. We use $S\left(x, \theta_{i}\right)$ to denote a bidder's bid on quantity $x$ when he is of type $\theta_{i}$. Throughout the paper, we denote utility and bidding functions with capital letters and denote the respective derivatives with small letters. For example, we write $U_{i}$ for the utility function and $u_{i}$ for marginal utility. Also, $\underline{U}=U(\cdot, \underline{\theta})$ denotes the utility function of the weakest possible bidder.

We can now formally define what truthful bidding in the clock means. A bidder bids truthfully at clock price $p$ if the demanded share $x_{i}(p)$ is such that $u_{i}\left(x_{i}(p)\right)=p$. We say that a bidder expands demand in the clock phase if there are clock prices such that the bidder demands an amount $x_{i}$ with $u_{i}\left(x_{i}\right)<p$. As marginal utilities are decreasing in $x_{i}$, it is clear that this inequality can only hold if bidders demand more than their truthful demand. A bidder reduces demand at clock price $p$ if $u_{i}\left(x_{i}(p)\right)>p$.

Figure 1 illustrates an important aspect of the activity rules. In the left panel, a bidder has continuously decreased his demand in the clock phase until the clock stops at price $\tilde{p}$ where he demands $\tilde{x}$. Given this clock behavior, the shaded area under the demand curve represents the maximal amount bidder $i$ can bid in the supplementary round on the full supply relative to his supplementary bid on $\tilde{x}$. As we show in more detail in the next section, this area is essential to understanding the extent to which a bidder can raise his rival's costs. Now suppose, as represented in the right panel, that the bidder continues bidding on the full supply until price $\tilde{p}$ and then drops the demand to $\tilde{x}$. In this case, the size of the shaded area is larger, namely $\tilde{p}(1-\tilde{x})$, so that the bidder potentially is able to raise his rival's costs further. As explained in the Introduction, in their third model, Levin and Skrzypacz (2016) do not take these constraints of the activity rules into account and assume that bidders use linear bid strategies (as in the left panel), but can raise their rival's costs in the supplementary round as if their clock behavior is as in the right panel. 
Bidders use the information about the clock development to update their beliefs about the type of the rival bidder. Even though no information about demand is revealed, bidders can infer information about their competitor's type from the equilibrium strategies and the duration of the clock phase. We denote by $\Theta$ the support of the posterior of the other bidder's type distribution. In a pooling equilibrium, a bidder does not learn anything about the other bidder's type, so $\Theta=[\underline{\theta}, \bar{\theta}]$. On the contrary, the equilibrium might be separating so that the final clock price reveals the rival's type. The posterior in such an equilibrium is then the singleton $\boldsymbol{\Theta}=\left\{\theta_{j}\right\}$. The set $\boldsymbol{\Theta}(p)$ denotes the support of the posterior if the clock ends at price $p$.

In addition to the standard preferences, bidders have a spite motive. We model this spite motive in a lexicographic way. In the first dimension, each bidder maximizes his surplus from the auction, and in the second dimension, he maximizes the payment of the other bidder. We sometimes refer to the (standard) expected utility of the first dimension as the primary utility. The spite motive is relatively weak since bidders do not want to harm the other bidder if this implies getting a lower surplus themselves.

For the VCG auction, we define the preference for raising the rival's costs as follows. Given the other bidder's strategy $S_{j}$, bidder $i$ strictly prefers strategy $\hat{S}_{i}$ over strategy $S_{i}$ if and only if $\hat{S}_{i}$ yields a strictly higher primary expected utility than $S_{i}$, or the primary expected utility is the same and $\hat{S}_{i}$ leads to a weakly higher VCG price for bidder $j$ for all $\theta_{j} \in[\underline{\theta}, \bar{\theta}]$ and to a strictly higher VCG price for a least one $\theta_{j} \in[\underline{\theta}, \bar{\theta}]$. More formally, let $\hat{\mathbf{x}}(\theta)$ be the allocation implemented by $\left(\hat{S}_{i}, S_{j}\right)$ and let $\mathbf{x}(\theta)$ be the allocation implemented by the strategy profile $\left(S_{i}, S_{j}\right)$. The strategy $\hat{S}_{i}$ is preferred to $S_{i}$ in the spite dimension if

$$
\max _{y} \hat{S}_{i}(y)-\hat{S}_{i}\left(\hat{x}_{i}(\theta)\right) \geq \max _{y} S_{i}(y)-S_{i}\left(x_{i}(\theta)\right)
$$

for all $\theta_{j} \in[\underline{\theta}, \bar{\theta}]$ with a strict inequality for a least one $\theta_{j} \in[\underline{\theta}, \bar{\theta}]$.

For the CCA, the definition of raising the rival's costs has to be slightly adapted as follows. First, in the CCA, a strategy consists of a clock demand function $x_{i}$ and a supplementary bidding function $S_{i}^{p}$ for every possible final clock price $p$. Accordingly, the VCG strategy $S_{i}$ has to be replaced by the CCA strategy $\left(x_{i},\left\{S_{i}^{p}\right\}_{p}\right)$. Second, the dynamic nature of the CCA needs to be taken into account. A strategy $\left(\hat{x}_{i},\left\{\hat{S}_{i}^{p}\right\}_{p}\right)$ is then weakly preferred to another strategy $\left(x_{i},\left\{S_{i}^{p}\right\}_{p}\right)$ if for any history of the clock phase, the continuation strategy is weakly preferred. Conditional on the clock price $\tilde{p}$ being reached, the difference with the VCG mechanism is that we use the posterior $\boldsymbol{\Theta}(\tilde{p})$ rather than the prior belief $[\underline{\theta}, \bar{\theta}]$ to evaluate a bidder's preference. This means that if the clock ended at price $\tilde{p}$, the supplementary bidding function $\hat{S}_{i}^{\tilde{p}}$ is weakly preferred to $S_{i}^{\tilde{p}}$. When the clock has not ended at price $\tilde{p}$, then the evaluation of whether the continuation strategy $\left(\left.\hat{x}_{i}\right|_{p \geq \tilde{p}},\left\{\hat{S}_{i}^{p}\right\}_{p \geq \tilde{p}}\right)$ is weakly preferred to $\left(\left.x_{i}\right|_{p \geq \tilde{p}},\left\{S_{i}^{p}\right\}_{p \geq \tilde{p}}\right)$ again uses the posterior $\Theta(\tilde{p})$ and not the prior.

Efficiency and equilibrium For every type profile $\theta$, we define the efficient allocation $\mathbf{x}^{*}=\left(x_{1}^{*}, x_{2}^{*}\right)$ as $\mathbf{x}^{*}(\theta) \in \arg \max _{x} U\left(x_{1}, \theta_{1}\right)+U\left(x_{2}, \theta_{2}\right)$ such that $x_{1}+x_{2} \leq 1$ and $x_{i} \geq 0$ for $i=1,2$. 
A few results are immediate. Since the utility functions are strictly increasing and concave, there exists a unique efficient allocation, which may involve one bidder not getting anything. As the objective function of the constrained maximization problem is supermodular in $\left(x_{i}, \theta_{i}\right)$, Topkis's monotonicity theorem implies that bidder $i$ 's efficient share $x_{i}^{*}\left(\theta_{i}, \theta_{j}\right)$ is nondecreasing in $\theta_{i}$ and, hence, it is nonincreasing in $\theta_{j}$. It follows that

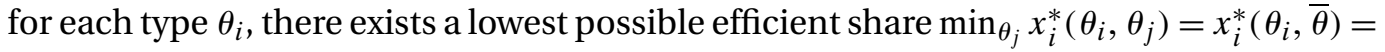
$\underline{x}_{i}$ and a largest possible efficient share $\max _{\theta_{j}} x_{i}^{*}\left(\theta_{i}, \theta_{j}\right)=x_{i}^{*}\left(\theta_{i}, \underline{\theta}\right)=\bar{x}_{i}$. Concavity of $U$ implies that the allocation $(1 / 2,1 / 2)$ is efficient for any symmetric type profile. As a consequence, for types $\underline{\theta}<\theta_{i}<\bar{\theta}$, we have that $\underline{x}_{i}<1 / 2<\bar{x}_{i}$. In any efficient allocation, the lowest type never wins more than $1 / 2$, while the strongest type $\bar{\theta}$ does not win less than $1 / 2$. Berge's maximum theorem implies that $\mathbf{x}^{*}(\theta)$ is continuous in $\theta$. Hence, for any $x \in\left[\underline{x}_{i}, \bar{x}_{i}\right]$, there exists a type $\theta_{j}$ such that $(x, 1-x)=\mathbf{x}^{*}(\theta)$. Finally, we note that $u\left(\bar{x}\left(\theta_{i}\right), \theta_{i}\right)$ is nondecreasing in $\theta_{i} .{ }^{11}$ The value function of the maximization problem defining the efficient allocation is $V(\theta)=U_{1}\left(x_{1}^{*}\right)+U_{2}\left(x_{2}^{*}\right)$. It is nondecreasing in $\theta_{i}$ for all $i$, since by the envelope theorem, $\partial V(\theta) / \partial \theta_{i}=\partial U\left(x_{i}^{*}, \theta_{i}\right) / \partial \theta_{i} \geq 0$. We denote the minimal value of the efficient allocation when bidder $i$ has type $\theta_{i}$ by $V_{i}(\underline{\theta})=V\left(\theta_{i}, \underline{\theta}\right)$.

We consider weakly clock-monotone equilibria, i.e., equilibria where $\theta_{i} \geq \theta_{j} \Rightarrow$ $x_{i}(p) \geq x_{j}(p)$. Our equilibrium concept is a refinement of the ex post equilibrium applied to the first dimension of the preferences. We consider only ex post equilibria that are such that given the prior beliefs and the strategies of the others, no bidder prefers to use a different strategy as defined above, including the preference for raising his rival's costs. We cannot use the notion of ex post equilibrium using the full preferences, as in equilibrium we must allow for the fact that knowing the type of the competitor ex post, a bidder may want to change the rival's cost by raising bids.

Quadratic utility model Levin and Skrzypacz (2016) consider a particular instance of our model, where bidders have a strictly increasing quadratic utility function of the form

$$
U\left(x, \theta_{i}\right)=\theta_{i} x-\frac{\sigma}{2} x^{2},
$$

with $\underline{\theta} \geq \sigma>0$ and $x \in[0,1]$. The condition $\underline{\theta} \geq \sigma$ makes the utility function increasing in $x$ for all types. Levin and Skrzypacz (2016) adopt the assumption that $\bar{\theta}-\underline{\theta}<\sigma$, which guarantees that the efficient allocation is always in the interior of $[0,1]$ as $u_{i}(0)>u_{j}(1)$, $j \neq i$. The efficient share of bidder $i$ is then

$$
x_{i}^{*}\left(\theta_{i}, \theta_{j}\right)=\frac{\theta_{i}-\theta_{j}+\sigma}{2 \sigma} .
$$

We use the quadratic utility function in our examples. Note that in our general setup, we do not assume that the efficient allocation is always in the interior.

\footnotetext{
${ }^{11}$ This can be seen as follows. Let $\theta_{i}^{\prime}>\theta_{i}$, so $\bar{x}_{i}^{\prime} \geq \bar{x}_{i}$. Suppose $\bar{x}_{i}^{\prime}=\bar{x}_{i}=1$. Then clearly $u\left(1, \theta_{i}^{\prime}\right)>u\left(1, \theta_{i}\right)$. If $\bar{x}_{i}^{\prime}=1$, but $\bar{x}_{i}<1$, then $u\left(1, \theta_{i}^{\prime}\right) \geq u(0, \underline{\theta}) \geq u\left(1-\bar{x}_{i}, \underline{\theta}\right)=u\left(\bar{x}_{i}, \theta_{i}\right)$ by decreasing marginal values and necessary conditions of efficiency. If $1>\bar{x}_{i}^{\prime}$, then efficiency requires $u\left(\bar{x}_{i}, \theta_{i}\right)=u\left(1-\bar{x}_{i}, \underline{\theta}\right)$ and $u\left(\bar{x}_{i}^{\prime}, \theta_{i}^{\prime}\right)=$ $u\left(1-\bar{x}_{i}^{\prime}, \underline{\theta}\right)$. Since $1-\bar{x}_{i} \geq 1-\bar{x}_{i}^{\prime}$, decreasing marginal values imply $u\left(1-\bar{x}_{i}, \underline{\theta}\right) \leq u\left(1-\bar{x}_{i}^{\prime}, \underline{\theta}\right)$.
} 


\section{EFFICIENCY AND INFORMATION REVELATION}

This section presents the fundamental trade-off between efficiency and information revelation. Figure 1 has shown how bidders can expand demand in the clock phase to relax the constraints imposed by the activity rule to raise their rival's cost in the supplementary round. Whether a bidder fully wants to use the potential to raise the bid on the full supply depends on his knowledge of the competitor's type. Bidding high on the full supply can be risky if one does not know how strong the competitor is. Learning the competitor's type allows a bidder to increase his rival's CCA price by allowing the bidder to target more precisely the amount by which he can raise the bid on the full supply without acquiring it.

Knowing how bidders can relax the constraints imposed by the activity rule to be able to raise their rival's cost in the supplementary round, we now consider how this ability eliminates the possibility of fully revealing efficient equilibria, i.e., equilibria where at the end of the clock phase bidders know the type of their competitor. Full revelation requires that the clock phase bidding strategies are separating. Truthful bidding is an example of a separating clock phase bidding strategy.

The impossibility of having fully revealing efficient equilibria can be explained along the following lines. First, full revelation and efficiency together require that on all possibly efficient shares $\left[\underline{x}_{i}, \bar{x}_{i}\right]$, all bidders must bid truthfully in the clock phase and the clock phase ends with market clearing. Thus, for all prices $p \in\left[u_{i}\left(\bar{x}_{i}\right), u_{i}\left(\underline{x}_{i}\right)\right]$ demand should be such that $u_{i}\left(x_{i}\right)=p$. For any other fully revealing clock bidding strategy, at least some types cannot express their true marginal utility in the auction. Second, market clearing in the final clock round implies that the final clock round demands are equal to the final allocation. The supplementary round bids then only determine the price the competitor has to pay. Third, given such a fully revealing strategy of their competitor, weak types have an incentive to deviate and expand demand at least until the clock price is in the interior of this interval and then drop demand discontinuously and demand truthfully from then on. This deviating strategy is illustrated in Figure 2, where, in the left panel, truthful demand is given by the solid line over the interval $p \in\left[u_{i}\left(\bar{x}_{i}\right), u_{i}\left(\underline{x}_{i}\right)\right]$ and the dashed line indicates that outside this interval demand can be anything as long as it is weakly decreasing. The middle panel of Figure 2 depicts the deviation strategy that is considered, where the bidder expands demand for prices $p \in\left[u_{i}\left(\bar{x}_{i}\right), p^{\prime}\right)$ for some $p^{\prime} \in\left(u_{i}\left(\bar{x}_{i}\right), u_{i}\left(\underline{x}_{i}\right)\right]$ and bids truthfully for all $p \in\left[p^{\prime}, u_{i}\left(\underline{x}_{i}\right)\right]$. Note that the nondeviating bidder does not notice the deviation, as $p^{\prime}$ is on the equilibrium path so that after the clock stops at $p^{\prime}$, he simply believes that the rival is of a stronger type.

To see that this deviation is beneficial, two cases should be distinguished. First, suppose that the competitor is of a relatively strong type and that under truthful bidding the clock phase would stop at a price $p \in\left[p^{\prime}, u_{i}\left(\underline{x}_{i}\right)\right]$. In this case, the deviation goes unnoticed by the competitor, the final clock price would remain unchanged, and the clock ends with market clearing. However, the deviation weakens the constraints imposed by the activity rules and allows the deviating bidder to raise his rival's costs beyond what would be possible if he had not deviated. Second, suppose that the competitor is of a relatively weak type and that under truthful bidding the clock phase would stop at a 
— Clock Demand for $p \in\left[u_{i}\left(\bar{x}_{i}\right), u_{i}\left(\underline{x}_{i}\right)\right]$

-- Marginal Supp. Bids for $x \in\left[x_{i}\left(p^{\prime}\right), \bar{x}_{i}\right]$
- - (Possible) Clock Dem. for $p \notin\left[u_{i}\left(\bar{x}_{i}\right), u_{i}\left(\underline{x}_{i}\right)\right]$

Relative Cap for $x \in\left(x_{i}\left(p^{\prime}\right), \bar{x}_{i}\right]: s_{i}(x) \leq p^{\prime}$

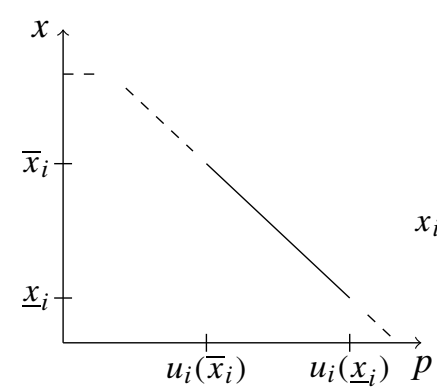

(a) Truthful bidding for $p \in\left[u_{i}\left(\bar{x}_{i}\right), u_{i}\left(\underline{x}_{i}\right)\right]$

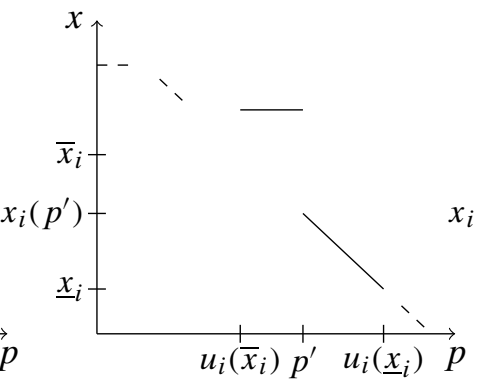

(b) Demand expansion for $p \in\left[u_{i}\left(\bar{x}_{i}\right), p^{\prime}\right)$

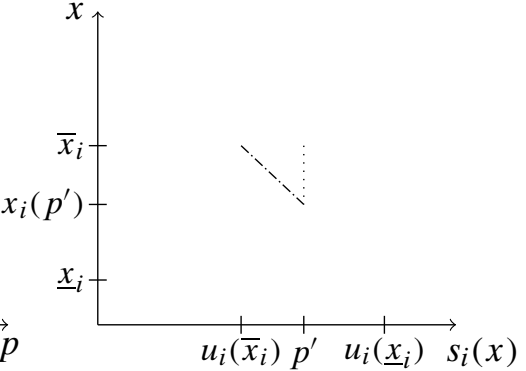

(c) Marginal supplementary bids when clock phase ends at $p^{\prime}$ after demand expansion

Figure 2. Profitable demand expansion given truthful bidding

price $p \in\left[u_{i}\left(\bar{x}_{i}\right), p^{\prime}\right)$. In this case, after expanding demand, the clock phase immediately stops at price $p^{\prime}$ with excess supply. The competitor (mistakenly) infers from the final clock price $p^{\prime}$ that the deviating bidder is a stronger type than his true type. The deviating bidder can correct his deviation in the supplementary round by generating "missing" truthful bids in the interval $\left(x_{i}\left(p^{\prime}\right), \bar{x}_{i}\right]$. This is illustrated in the right panel of Figure 2, where marginal supplementary bids of the deviating bidder are depicted. The bidder bids true marginal utilities over the whole interval of possibly efficient shares $\left(x_{i}\left(p^{\prime}\right), \bar{x}_{i}\right]$ (depicted by the dotted-dashed line segment). The dotted line represents the constraints of the relative cap, which require that the solid line is to the left of the dotted line. This supplementary demand where bidder $j$ bids true marginal values is consistent with all activity rules. Hence, the same allocation is implemented as under truthful bidding and the deviating bidder pays the same price. Thus, the bidder is better off against some types and not worse off against other types, making the deviation beneficial.

The following proposition states the result formally and the proof explains the argument in more (technical) detail. We use the function $\tau:[\underline{\theta}, \bar{\theta}]^{2} \rightarrow \mathbb{R}_{+}$to analyze equilibrium information revelation. The function assigns, for a given equilibrium, to every type profile the final equilibrium clock price, i.e., $\tau(\theta)=\inf \left\{p: x_{1}(p)+x_{2}(p) \leq 1\right\}$. If no information is revealed during the clock phase and the clock ends at the same price for all type profiles, there exists a price $p$ such that $\tau(\theta)=\{p\}$ for all type profiles $\theta \in[\underline{\theta}, \bar{\theta}]^{2}$. We call this a clock-pooling equilibrium. At the other extreme case is a clock-separating equilibrium, which is defined as an equilibrium where the function $\tau(\theta)$ is nondecreasing in $\theta_{i}$ for all $i$ and strictly increasing in $\theta_{j}$ for all bidders $j$ who win a share $x_{j}<1$. $^{12}$

\footnotetext{
${ }^{12}$ The restriction $x_{j}<1$ is needed for the following reason. Suppose $[\underline{\theta}, \bar{\theta}]$ is such that a bidder $i$ with type $\underline{\theta}$ wins 0 in the efficient allocation if bidder $j$ 's type is sufficiently close to $\bar{\theta}$. In this case, the clock phase would stop at the same clock price for all bidders $j$ who win the full supply.
} 
Proposition 1. There does not exist an efficient clock-separating equilibrium in the CCA.

An immediate implication of the proposition is that truthful bidding is not an equilibrium. In addition, if one interprets a clock-separating equilibrium as a formal definition of the more informal notion of price and package discovery, mentioned in the Introduction (e.g., Ausubel et al. 2006), then it follows that under a weak preference for raising a rival's costs, the CCA cannot deliver its two main objectives simultaneously: efficiency and price and package discovery.

The result is akin to the ratchet effect in the dynamic contracting literature (Laffont and Tirole 1988). In this literature, high types do not want to reveal their type in the first period, because they would end up with a worse contract in the second period if their type is revealed. In the CCA, the driving forces are similar. Suppose a low type observes that the clock has not ended yet, indicating that the other bidder has a high type. A low type can use this information to raise the high type's CCA price if the constraints of the activity rule are not binding, which is the case if the low type has expanded demand for a sufficiently long period. Higher types will then best respond by pooling with lower types so as to obfuscate their type.

We now argue that Proposition 1 continues to hold if, in the supplementary phase, bidders are informed about the final clock round (individual or aggregate) demand. The proof of Proposition 1 shows that in an efficient and fully revealing equilibrium, bidders must bid according to true marginal values for clock prices $p \in\left[u_{i}\left(\bar{x}_{i}\right), u_{i}\left(\underline{x}_{i}\right)\right]$ and the clock will end with market clearing. This argument is independent of the information policy. The difference between whether bidders are informed about the demand in the final clock round is that without demand disclosure, a deviation to further demand expansion will not be noticed by the nondeviating bidder, whereas with demand disclosure, the deviation will be detected when the clock ends with excess supply. To understand that Proposition 1 continues to hold with demand disclosure, it is important to realize that the nondeviating bidder learns about the deviation only when the clock phase ends, so his supplementary bids are constrained by the relative cap in the same way as without demand disclosure. It then suffices to show that there are deviations in the clock phase such that the nondeviating bidder has to bid true marginal values on relevant shares in the supplementary round after the clock ends with excess supply. In this case, the deviating bidder can correct his clock bids in the supplementary phase and as the nondeviating bidder cannot further raise his rival's cost given his clock demand, the deviating bidder is able to implement the same allocation but raise his rival's cost.

To finish the argument, we now show that there are (out-of-equilibrium) clock phase endings with excess supply where the nondeviating bidder $j$ must bid true marginal values in the supplementary phase. Consider the clock ending at $\tilde{p}=u_{j}\left(\bar{x}_{j}\right)+\epsilon$ with excess supply and supplementary bids on $\left[x_{j}(\tilde{p}), \bar{x}_{j}\right]$ with $x_{j}(\tilde{p})$ being type $j$ 's truthful demand at $\tilde{p}$, i.e., $\tilde{p}=u_{j}\left(x_{j}(\tilde{p})\right)$. To have a fully revealing, efficient ex post equilibrium, the following strategy for all types $\theta_{i}$ with $1-\bar{x}_{j} \leq x^{*}\left(\theta_{i}, \theta_{j}\right)<1-x_{j}(\tilde{p})$ must not be a profitable deviation: in the clock phase, demand 1 for prices less than $\tilde{p}$, and demand $x^{*}\left(\theta_{i}, \theta_{j}\right)$ at $\tilde{p}$ and in the subsequent supplementary phase bid only on the efficient share and the 
full supply. It is clear that after such a deviation, the efficient allocation is implemented. To make this a nonprofitable deviation, it should be the case that bidder $i$ pays a price that is not smaller than the price he has to pay after the clock ends with market clearing and truthful bidding. Note, however, that the postdeviation CCA price cannot be higher, as bidder $j$ already fully raises the CCA price on the equilibrium path. Bidder $j$ must, therefore, bid true marginal values on $\left[x_{j}(\tilde{p}), \bar{x}_{j}\right]$ when the clock ends with excess supply at $\tilde{p}$ with demands $\left(x_{i}^{*}, \tilde{x}_{j}\right)$. Consequently, as in the proof of Proposition 1, bidder $i$ can demand 1 for prices less than $\tilde{p}$ and demand truthfully at $\tilde{p}$, where truthful demand is $x_{i}(\tilde{p})<x^{*}\left(\theta_{i}, \theta_{j}\right)$, and bid true marginal values in the subsequent supplementary phase. The deviation increases the rival's cost without affecting the allocation and his own payment.

The next proposition states a property of any efficient equilibrium, namely that the clock cannot stop at very low prices and that weak bidders expand demand at some stage of the clock phase. In combination with the next subsection where we construct an efficient equilibrium, this proposition is of interest as it shows that, in contrast to Levin and Skrzypacz (2016), bidders do not necessarily want to reduce demand in the face of a competitor with a spite motive. The example of an efficient equilibrium also shows that the fact that efficient clock-separating equilibria do not exist in either information regime does not mean that efficient equilibria do not exist in general.

Proposition 2. In any efficient equilibrium, a bidder will not demand $\hat{x}_{i} \leq \bar{x}_{i}$ at prices $p<\min \left\{u(1, \bar{\theta}), u_{i}\left(\hat{x}_{i}\right)\right\}$. The smallest final clock price $\tilde{p}$ is strictly larger than $\min \{u(1, \bar{\theta}), u(1 / 2, \underline{\theta})\}$ and some types expand demand for some prices $p<\tilde{p}$.

The argument is as follows. Suppose to the contrary that in an efficient equilibrium, bidder $i$ demands $\hat{x}_{i} \leq \bar{x}_{i}$ at clock price $p<\min \left\{u(1, \bar{\theta}), u_{i}\left(\hat{x}_{i}\right)\right\}$. We distinguish two cases. First, suppose $\hat{x}_{i}$ is possibly an efficient share, i.e., $\underline{x}_{i} \leq \hat{x}_{i} \leq \bar{x}_{i}$. Efficiency of equilibrium requires that bidders bid true marginal values on possible efficient shares. However, this is not feasible for bidder $i$, as, independent of whether the clock ends at $p$ or continues, the relative cap imposes that the supplementary bids for $\hat{x}_{i}$ must satisfy $s_{i}\left(\hat{x}_{i}\right) \leq p<u_{i}\left(\hat{x}_{i}\right)$. Hence, in an efficient equilibrium where bidders reduce demand at these low prices, it must be that $\hat{x}_{i}<\underline{x}_{i}$.

Second, we argue that in an ex post equilibrium, the highest types do not want to implement the efficient allocation if their competitor reduces demand to $\hat{x}_{i}<\underline{x}_{i}$. Consider bidder $j$ with type $\theta_{j}=\bar{\theta}$. In an efficient ex post equilibrium, in the supplementary phase in which bidders $i$ and $j$ meet, bidder $j$ must prefer winning the efficient share $x_{j}^{*}$ over $1-\hat{x}_{i}$, i.e.,

$$
U_{j}\left(1-\hat{x}_{i}\right)-\max _{y} S_{i}(y)+S_{i}\left(\hat{x}_{i}\right) \leq U_{j}\left(1-x_{i}^{*}\right)-\max _{y} S_{i}(y)+S_{i}\left(x_{i}^{*}\right) .
$$

As $x_{i}^{*}>\hat{x}_{i}$, the relative cap implies that $S_{i}\left(x_{i}^{*}\right) \leq S_{i}\left(\hat{x}_{i}\right)+p\left(1-x_{j}^{*}-\hat{x}_{i}\right)$ so that the above inequality implies

$$
U_{j}\left(1-\hat{x}_{i}\right)-U_{j}\left(x_{j}^{*}\right) \leq p\left(1-\hat{x}_{i}-x_{j}^{*}\right) .
$$


However, as $p \leq u(1, \bar{\theta})$, this inequality cannot hold, i.e., the strongest types of bidder $j$ strictly prefer winning $1-\hat{x}_{i}$ over the efficient share.

Given this argument and the impossibility of fully revealing equilibria, it is clear that the clock cannot stop at a price $\tilde{p} \leq \min \{u(1, \bar{\theta}), u(1 / 2, \underline{\theta})\}$. As at least one bidder must demand less than $1 / 2$ for the clock to end, it is clear that at these relatively low clock prices this bidder reduces demand and to a quantity smaller than $\bar{x}_{i}$, which we have just shown is not possible in an efficient equilibrium. It is then also easy to see that some bidders would want to expand demand at some prices $p \leq \tilde{p}$. Doing so, while keeping fixed the rest of the clock phase bidding, allows the bidder to raise the rival's cost in the supplementary round in a way that does not risk winning these bids, as explained above.

\subsection{Efficient equilibria in the quadratic utility model}

Proposition 1 rules out fully revealing efficient equilibria. In this subsection, we present an example where bidders have quadratic utility functions. The example shows that (i) equilibria exist, (ii) equilibria can be efficient, and (iii) what equilibria with demand expansion in the first phase of the clock may look like. Thus, the previous propositions have economic content and are not due to a lack of equilibrium existence. The example is also useful to understand the main intuition behind the second main result presented in the next section.

The equilibrium is clock semi-separating as the bidding in the clock phase might reveal some information about the rival's type. Both bidders expand demand by bidding on the full supply until a threshold clock price $\tilde{p}>u(1 / 2, \underline{\theta})$. At prices larger than the threshold price, bidders bid truthfully. In accordance with the previous propositions, as $\tilde{p}>u(1 / 2, \underline{\theta})$ and bidders bid truthfully for prices $p \geq \tilde{p}$, there are (at least) some low types for which there is pooling behavior in the clock phase. The threshold price plays a crucial role in the equilibrium construction, and we will identify constraints on it for this type of equilibrium to exist.

Clock behavior As bidders have quadratic utility functions, the above described clock behavior with extreme demand expansion at prices $p<\tilde{p}$ and bidding according to true marginal values for $p \geq \tilde{p}$ gives clock demand

$$
x_{i}(p)= \begin{cases}1 & \text { if } p<\tilde{p} \\ \max \left\{\frac{\theta_{i}-p}{\sigma}, 0\right\} & \text { if } p \geq \tilde{p} .\end{cases}
$$

Figure 3 illustrates the two possible ways in which the clock can end in equilibrium: the clock ends either (i) with excess supply at $\tilde{p}$ or (ii) with market clearing at $p^{*}>\tilde{p}$. The figure shows bidder 1's clock demand function (the dashed line) and $1-x_{2}(p)$, the residual supply function faced by bidder 1 (the solid line). The dotted shaded (line shaded) area between the two curves at price $p$ indicates excess demand (supply). In the two plots, bidder 1's demand is the same. Bidder 2's type determines whether the left or the right figure applies to the clock phase. If bidder 2's type is sufficiently low, aggregate demand at $\tilde{p}$ is smaller than the available supply (Figure 3(a)). Conversely, when bidder 


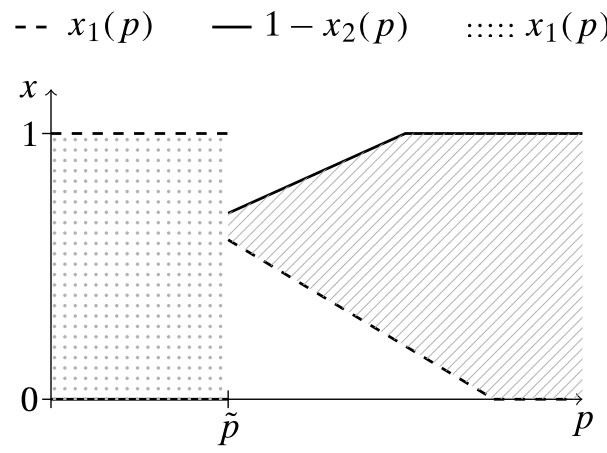

(a) Clock ends at $\tilde{p}$$$
\text { III. } x_{1}(p)+x_{2}(p)<1
$$

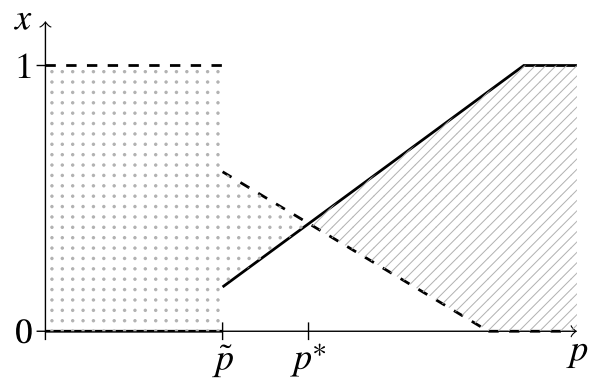

(b) Clock ends at $p^{*}>\tilde{p}$

FIgURE 3. Clock behavior in the semi-separating equilibrium.

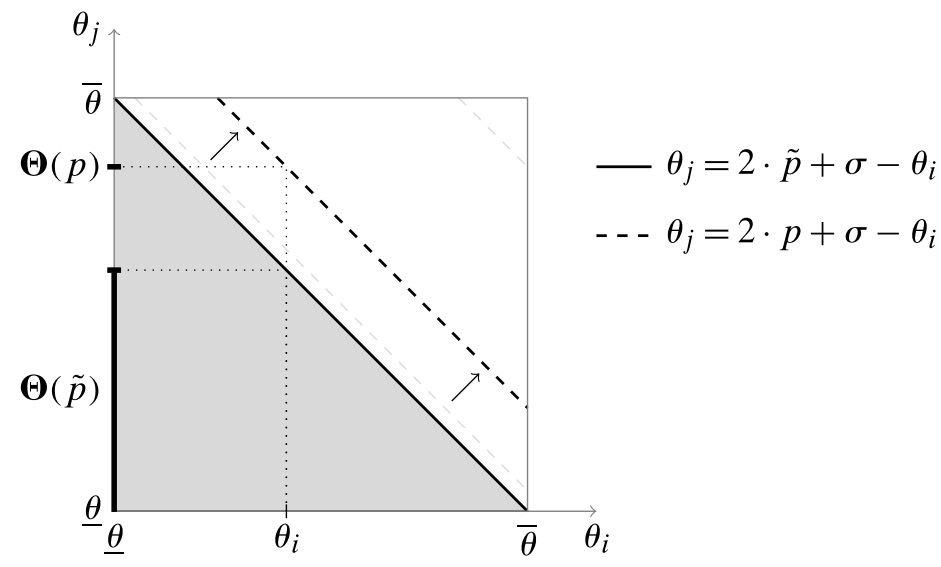

FIGURE 4. Information revelation in the semi-separating equilibrium for $\tilde{p}=(\underline{\theta}+\bar{\theta}-\sigma) / 2$.

2's type is high, there may be excess demand at $\tilde{p}$ (Figure 3(b)). Clearly, we must have $\tilde{p}<\bar{\theta}-\sigma / 2$ so that the highest types demand more than half of the supply at $\tilde{p}$. At $p>\tilde{p}$, bidders bid truthfully and the clock eventually ends with market clearing at $p^{*}>\tilde{p}$.

Bidders update their prior about the other bidder as the clock proceeds. Figure 4 summarizes the information revelation during the course of the clock phase. The square depicts all possible type profiles. The clock ends at $\tilde{p}$ for type profiles in the gray area, i.e., if $\theta_{i}+\theta_{j} \leq 2 \tilde{p}+\sigma$. Hence, bidder $i$ with type $\theta_{i}$ infers from the clock ending at $\tilde{p}$ that $j$ 's type is at most $2 \tilde{p}+\sigma-\theta_{i}$, i.e., $\boldsymbol{\Theta}(\tilde{p})=\left[\underline{\theta}, 2 \tilde{p}+\sigma-\theta_{i}\right]$. If the types are such that the clock does not stop at $\tilde{p}$, the parallel diagonal lines reflect the combination of types for which the clock ends at $p>\tilde{p}$. For each such $p$, the clock ends with market clearing for types $\left(\theta_{i}, \theta_{j}\right)$ such that $\left(\theta_{i}-p\right) / \sigma+\left(\theta_{j}-p\right) / \sigma=1$, yielding the lines $\theta_{j}=2 p+\sigma-\theta_{i}$. As the clock proceeds, the diagonal line in Figure 4 shifts to the northeast. If the clock does not stop at $\tilde{p}$, bidder $i$ knows that at any $p>\tilde{p}$, the lowest possible type of the other bidder is $2 p+\sigma-\theta_{i}$. Observing the final clock price $p^{*}$, each type $\theta_{i}$ correctly infers the rival's type $\boldsymbol{\Theta}\left(p^{*}\right)=\left\{2 p^{*}+\sigma-\theta_{i}\right\}$ in the candidate equilibrium. 
Supplementary bids The supplementary bidding functions depend on whether the clock ends at $\tilde{p}$ or at $p^{*}$. If the clock ends at $\tilde{p}$, bidder $i$ bids in the supplementary phase according to

$$
S_{i}^{\tilde{p}}(x)= \begin{cases}0 & \text { for } x<\tilde{x}_{i} \\ U_{i}(x) & \text { for } \tilde{x}_{i} \leq x<1 \\ \min \left\{U_{i}\left(\bar{x}_{i}\right)+\underline{U}\left(1-\bar{x}_{i}\right), U_{i}\left(\tilde{x}_{i}\right)+\tilde{p}\left(1-\tilde{x}_{i}\right)\right\} & \text { for } x=1,\end{cases}
$$

where $\tilde{x}_{i}$ is bidder $i$ 's truthful demand at $\tilde{p} .{ }^{13}$ Each bidder bids true utility on shares that might be obtained given the clock behavior and submits a spiteful bid on 1, which will be discussed below. If the clock ends at $p^{*}>\tilde{p}$, bidder $i$ uses the bidding function

$$
S_{i}^{p^{*}}(x)= \begin{cases}U_{i}(x) & \text { for } x \leq \tilde{x}_{i} \\ U_{i}\left(\tilde{x}_{i}\right) & \text { for } \tilde{x}_{i}<x<1 \\ U_{i}\left(\tilde{x}_{i}\right)+\tilde{p}\left(1-\tilde{x}_{i}\right) & \text { for } x=1 .\end{cases}
$$

One difference between the two bidding functions is that bidder $i$ bids true marginal values on (efficient) shares higher than $\tilde{x}_{i}$ after $\tilde{p}$, but not after $p^{*}$. We see below that this difference prevents $j$ from further expanding demand in the clock phase. Another difference is, as explained below, the bid on the full supply.

It is straightforward to check that the supplementary bidding functions implement the efficient allocation and that they satisfy the activity rules given the stipulated clock behavior.

We now argue that these supplementary bidding functions are optimal from the perspective of raising the rival's cost in that bidders want to raise their rival's cost as much as possible without running the risk of winning a bid inadvertently. Whatever bidders bid on their last clock round share in the supplementary round, the relative cap implies they can maximally bid $\tilde{p}\left(1-\tilde{x}_{i}\right)$ more on the full supply if the clock ends at $p^{*}$ or $\tilde{p}$. However, when the clock phase ends at $\tilde{p}$ and their bid on the entire supply is more than $S_{i}\left(\bar{x}_{i}\right)-S_{i}\left(\tilde{x}_{i}\right)+\underline{S}\left(1-\bar{x}_{i}\right)$ higher than their bid on $\tilde{x}_{i}$, they run the risk of winning the full supply if the rival bidder's type is low. In the candidate equilibrium, this means that bidders do not want to bid more than $U_{i}\left(\bar{x}_{i}\right)+\underline{U}\left(1-\bar{x}_{i}\right)$ on the full supply. As a result, when the clock ends at $\tilde{p}$, bidders bid $S_{i}(1)=\min \left\{U_{i}\left(\bar{x}_{i}\right)+\underline{U}\left(1-\bar{x}_{i}\right), U_{i}\left(\tilde{x}_{i}\right)+\tilde{p}\left(1-\tilde{x}_{i}\right)\right\}$. Now we consider the clock ending at $p^{*}>\tilde{p}$. Observing the clock ended at $p^{*}$, bidders update their belief about the rival bidder's type and believe that the clock ended with market clearing. Due to the final cap rule, bidders believe that the final clock allocation is also the final allocation and maximally raise their bid on the full supply, i.e., for $x \in$ $\left[x_{i}\left(p^{*}\right), \tilde{x}_{i}\right]$ the relative cap $s_{i}(x) \leq u_{i}(x)$ holds with equality and $S_{i}(1)=S_{i}\left(\tilde{x}_{i}\right)+\tilde{p}\left(1-\tilde{x}_{i}\right)$.

For later reference, it is useful to consider how $U_{i}\left(\bar{x}_{i}\right)+\underline{U}\left(1-\bar{x}_{i}\right)$ and $U_{i}\left(\tilde{x}_{i}\right)+\tilde{p}(1-$ $\tilde{x}_{i}$ ) depend on a bidder's type. Both expressions are represented in Figure 5 . It turns out that there is a cutoff type

$$
\hat{\theta}(\tilde{p})=\tilde{p}(2+\sqrt{2})-\underline{\theta}(1+\sqrt{2})+\sigma
$$

\footnotetext{
${ }^{13}$ Bidding 0 on shares $x<\tilde{x}_{i}$ simplifies the proof that no bidder has an incentive to deviate.
} 


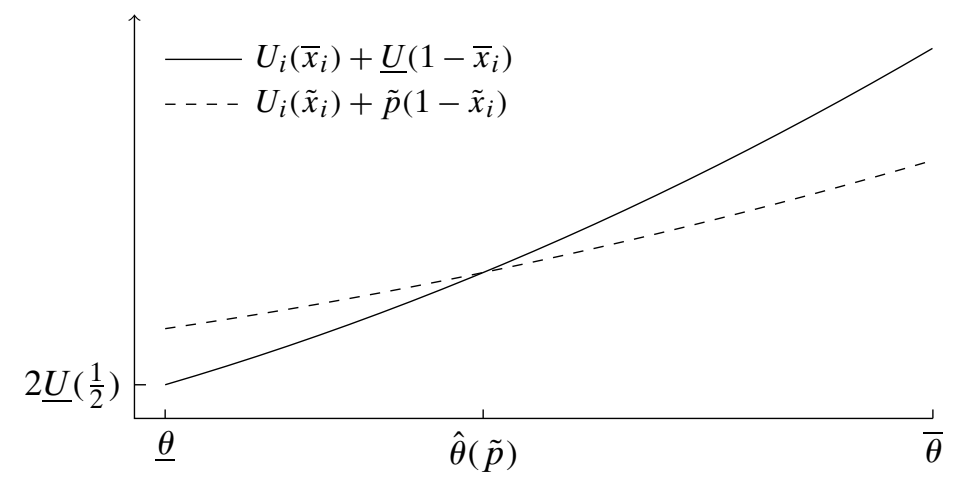

Figure 5. Constraints on the supplementary bid for the full supply.

such that $U_{i}\left(\bar{x}_{i}\right)+\underline{U}\left(1-\bar{x}_{i}\right)<U_{i}\left(\tilde{x}_{i}\right)+\tilde{p}\left(1-\tilde{x}_{i}\right)$ if and only if $\theta_{i}<\hat{\theta}(\tilde{p}) .{ }^{14}$ As a result, (only) bidders with a low type bid $S_{i}^{\tilde{p}}(1)=U_{i}\left(\bar{x}_{i}\right)+\underline{U}\left(1-\bar{x}_{i}\right)$.

Equilibrium constraints on clock behavior We now determine the restrictions on $\tilde{p}$ such that no bidder has an incentive to deviate in the clock phase. First, bidders should acquire positive utility from bidding. As the minimal value of the efficient allocation is attained if both bidders are of the lowest possible type, it is sufficient to require that $\underline{U}(1 / 2) \geq \tilde{p} / 2$, which is equivalent to $\underline{\theta}-\sigma / 4 \geq \tilde{p}$.

Second, it should not be the case that bidder $i$ wants to reduce his demand in the clock phase to prevent rival $j$ from raising the price $i$ has to pay if $j$ successively learns bidder $i$ 's type. To this end, define $\tilde{\theta}(\tilde{p})=2 \tilde{p}+\sigma-\bar{\theta}$ to be the highest type for which the clock always ends at $\tilde{p}$. Suppose now that $\hat{\theta}(\tilde{p})>\max \{\underline{\theta}, \tilde{\theta}(\tilde{p})\}$ so that there exists a type $\theta_{j} \in[\tilde{\theta}(\tilde{p}), \hat{\theta}(\tilde{p}))$ for which the clock phase does not necessarily stop at $\tilde{p}$ and the bidders' bid on the full supply is contingent on the final clock price. If the clock stops at $\tilde{p}$, they bid $U_{j}\left(\bar{x}_{j}\right)+\underline{U}\left(1-\bar{x}_{j}\right)$ as they do not want to risk winning the full supply. If the clock ends at a higher price, due to market clearing in the last clock round, they can safely bid $U_{j}\left(\tilde{x}_{j}\right)+\tilde{p}\left(1-\tilde{x}_{j}\right)$. Thus, for types in the interval $[\tilde{\theta}(\tilde{p}), \hat{\theta}(\tilde{p}))$, the clock not stopping at $\tilde{p}$ makes their bid on the full supply jump discretely by $U_{j}\left(\tilde{x}_{j}\right)+\tilde{p}\left(1-\tilde{x}_{j}\right)-\left(U_{j}\left(\bar{x}_{j}\right)+\underline{U}\left(1-\bar{x}_{j}\right)\right)$. Knowing this, it is profitable for some types higher than $\tilde{\theta}(\tilde{p})$-for whom the clock does not definitely stop-to reduce demand at $\tilde{p}$ to be certain to end the clock. Thus, $\hat{\theta}(\tilde{p})>\max \{\underline{\theta}, \tilde{\theta}(\tilde{p})\}$ cannot be part of an equilibrium. Alternatively, if $\tilde{\theta}(\tilde{p}) \geq \hat{\theta}(\tilde{p})$ or if $\hat{\theta}(\tilde{p}) \leq \underline{\theta}$, then the supplementary bids of all types $\theta_{j}>\tilde{\theta}(\tilde{p})$-for which the clock phase possibly continues at prices $p>\tilde{p}$-are independent of the final clock price, so that the bidders cannot raise their supplementary bids after obtaining information through the final clock price. For this case, we show that there is no incentive for demand reduction. Suppose type $\theta_{i}>\tilde{\theta}(\tilde{p})$ reduces demand at $\tilde{p}$. Let $\theta_{j}>2 \tilde{p}+\sigma-\theta_{i}$ be such that the clock ends at $\tilde{p}$ under $i$ 's demand reduction, although it would continue under truthful bidding. Bidder $j$ bids 0 on $x_{j}^{*}$ if the

\footnotetext{
${ }^{14}$ Formally, the equation $U_{i}\left(\bar{x}_{i}\right)+\underline{U}\left(1-\bar{x}_{i}\right)=U_{i}\left(\tilde{x}_{i}\right)+\tilde{p}\left(1-\tilde{x}_{i}\right)$ has a second $\operatorname{root} \hat{\theta}_{2}(\tilde{p})=\tilde{p}(2-\sqrt{2})+$ $\sigma-\underline{\theta}(1-\sqrt{2})$. As in all equilibria we consider, we have that $\tilde{p} \geq \min (\bar{\theta}-\sigma / 2, \underline{\theta}-\sigma / 4)$, so it is easy to see that $\overline{\hat{\theta}}_{2}(\tilde{p})>\bar{\theta}$ and that we effectively only have to consider $\hat{\theta}(\tilde{p})$.
} 
clock ends at $\tilde{p}$, as $x_{j}^{*}<\tilde{x}_{j}$. As the efficient allocation cannot be implemented, the demand reduction leads to a decrease in bidder $i$ 's primary utility. Therefore, equilibrium requires that $\tilde{\theta}(\tilde{p}) \geq \hat{\theta}(\tilde{p})$ or $\hat{\theta}(\tilde{p}) \leq \underline{\theta}$.

Third, we should also make sure that bidders do not have an incentive to expand demand further than is stipulated in the candidate equilibrium strategies by deviating and demanding more than $\tilde{x}_{i}(\tilde{p})$ until $p>\tilde{p}$. To this end, we first argue that for all types $\theta_{i}<\bar{\theta}$, the clock phase must stop at $\tilde{p}$ with positive probability in equilibrium. From the candidate equilibrium strategies, it is clear that if the clock can end for the highest possible type $\bar{\theta}$, it can also end for all other types. The reason the clock must possibly stop for all types is that if a bidder with type $\bar{\theta}$ knows that the clock will certainly not end under truthful bidding, then he prefers to continue demanding the full supply. To see this, recall that to have a semi-separating equilibrium, it should be the case that $\tilde{x}(\tilde{p}, \bar{\theta})>1 / 2$, which implies that $\tilde{p}<\bar{\theta}-\sigma / 2$. Given the definition of $\tilde{\theta}(\tilde{p})$ and the constraints derived in the previous paragraph, this implies that $\hat{\theta}(\tilde{p})<\bar{\theta}$. Thus, in the candidate equilibrium strategy, the activity rules restrain bidder $\bar{\theta}$ from fully raising the rival's cost. Continuing bidding on the entire supply would allow further raising the rival's cost without affecting the final allocation (and the price he pays). To make sure that it is possible for the clock to end along the equilibrium path for all types $\theta_{i}<\bar{\theta}$, it should be the case that $\tilde{p} \geq(\bar{\theta}+\underline{\theta}-\sigma) / 2=u(\bar{x}, \bar{\theta})=u(\underline{x}, \underline{\theta})$.

Next, we argue that expanding demand at $\tilde{p}$ results in a decrease of expected surplus, as there is a positive probability that the clock ends by bidding truthfully at $\tilde{p}$ for all types. To see this, we use the difference between the supplementary bidding strategies $S_{i}^{\tilde{p}}(x)$ and $S_{i}^{p^{*}}(x)$ in (1) and (2), respectively. If bidder $j$ were to bid truthfully, the clock would stop at $\tilde{p}$ for all types $\theta_{i} \leq 2 \tilde{p}+\sigma-\theta_{j}$. Importantly, the aggregate clock demand at $\tilde{p}$ is arbitrarily close to 1 if the competitor's type is just below $2 \tilde{p}+\sigma-\theta_{j}$. If the clock ends at $\tilde{p}$, we have $x_{j}^{*}>\tilde{x}_{j}$, so that the supplementary bidding function (1) guarantees that bidder $j$ gets the efficient share at a price $\min \left\{U_{i}\left(\bar{x}_{i}\right)+\underline{U}\left(1-\bar{x}_{i}\right), U_{i}\left(\tilde{x}_{i}\right)+\tilde{p}(1-\right.$ $\left.\left.\tilde{x}_{i}\right)\right\}-U_{i}\left(x_{i}^{*}\right)$. Consider then that bidder $j$ expands demand at $\tilde{p}$. In that case, there exist some types $\theta_{i}$ just below $2 \tilde{p}+\sigma-\theta_{j}$ for which the clock ends at a higher price than $\tilde{p}$. Given that the supplementary bid strategy of these types changes from (1) to (2), bidder $j$ gets at most a utility of $U_{j}\left(1-\tilde{x}_{i}\right)-\left[U_{i}\left(\tilde{x}_{i}\right)+\tilde{p}\left(1-\tilde{x}_{i}\right)-U_{i}\left(\tilde{x}_{i}\right)\right]$. As $1-\tilde{x}_{i}>x_{j}^{*}$ for some types $\theta_{i}$, bidder $j$ is better off not deviating.

All of the above constraints can be jointly satisfied for a variety of final clock prices. For example, we can set $\tilde{p}=(\bar{\theta}+\underline{\theta}-\sigma) / 2$ as we did in Figure 4 . At this price, the highest type for which the clock definitely ends at the threshold price is $\tilde{\theta}(\tilde{p})=\underline{\theta}$ and equilibrium exists whenever $\hat{\theta}(\tilde{p}) \leq \underline{\theta}$, or $\bar{\theta}-\underline{\theta} \leq(\sqrt{2}-1) \sigma$.

Discussion We conclude that a semi-separating equilibrium as discussed above exists if the uncertainty concerning the competitor's type, measured by $\bar{\theta}-\underline{\theta}$, is not too large. This equilibrium is efficient, as all bidders bid their true marginal utilities on possibly efficient shares in the supplementary phase and other bids are such that the winning bid combination is in this range of possibly efficient shares. Thus, there are efficient equilibria with some information revelation, where low types pool and high types are constrained by the activity rule so that they cannot exploit new information to raise their rival's cost. 
The semi-separating equilibrium is noteworthy as it shows that even if bidders know that the competitor is raising their cost in the supplementary phase, they do not reduce demand in the clock phase. This is in contrast to Levin and Skrzypacz (2016), who restrict bidders to linear proxy strategies and show that bidders will engage in demand reduction in the clock phase, assuming (against the auction rules) that a demand reduction strategy in the clock phase does not affect the ability to raise a rival's cost. The example also shows that, in contrast to what some observers of the CCA have argued, the clock phase may well end up with excess supply, while bidders are still able to raise their rival's cost. ${ }^{15}$

A variant of this equilibrium occurs if the clock stops at $\tilde{p}$ for all type profiles. In such a clock-pooling equilibrium, the clock does not reveal any information. There are three constraints on the clock-pooling price $\tilde{p}$. First, the truthful demand at $\tilde{p}$ of the strongest bidder should be smaller than half the supply for the clock to end. Second, no bidder should have an incentive to further expand demand because $\tilde{p}$ is such that the final cap and relative cap do not restrict the spiteful bid. Third, as before, the weakest type should still derive nonnegative utility. The constraints on $\tilde{p}$ specify a tighter upper bound on the range $\bar{\theta}-\underline{\theta}$ for such a clock-pooling equilibrium to exist.

\section{NONEXISTENCE OF EFFICIENT EQUILIBRIA WITH LARGE UNCERTAINTY}

So far we have seen that efficient clock-revealing equilibria do not exist, but that efficient equilibria may nevertheless exist even if bidders are spiteful. The example presented in the previous section constructs an efficient equilibrium where the uncertainty concerning the final allocations, measured by $\bar{\theta}-\underline{\theta}$, is relatively small. In this section, we consider auctions where the ex ante uncertainty concerning the final allocations is relatively large and, consequently, information revelation might be more important. Our second main result shows, however, that the CCA does not have efficient equilibria when the uncertainty about the other bidder's type is sufficiently large. To simplify the proof, we consider (type-) symmetric equilibria, that is, equilibria where identical types use identical strategies. ${ }^{16,17}$

\footnotetext{
${ }^{15}$ See, e.g., Levin and Skrzypacz (2016, Remark 2, p. 2542), where they observe that "[i]f we allowed bidder 2 to create excess supply at the end of the clock phase, she could increase bidder 1 payment even more.... Such extreme predatory behavior is even more difficult to execute and even more risky for player 2 than what we describe. Moreover, analyzing equilibria in this case is difficult, so we maintain the assumption that player 2 is not allowed to create excess supply in the clock phase." Similarly, Kroemer et al. (2016, p. 38) observe that "[i]n recent spectrum auction implementations, the regulator decided not to reveal excess supply in the last round, in order to make spiteful bidding risky. It depends on the market specifics, if this risk is high enough to eliminate spiteful bidding." The British regulator Ofcom (2015, A8.48 p. 16) also writes in a similar vein when they consider the Austrian 2013 CCA outcome: "We also noted that at the end of the clock rounds there was an excess supply of $2 \times 10 \mathrm{MHz}$ in each of the $900 \mathrm{MHz}$ and $1800 \mathrm{MHz}$ bands.... This further suggests a possible reason why bidders may have considered price driving in the supplementary bids to be a risky strategy...."

${ }^{16}$ We believe that asymmetric efficient equilibria do not exist either, but a formal proof would require checking many different cases.

${ }^{17}$ The proof of this result can also be used to show that efficient equilibria do not exist if the lowest type $\underline{\theta}$ does not value the good at all, i.e., $u(x, \underline{\theta})=0$.
} 
Proposition 3. Let $u(1, \bar{\theta})>u(0, \underline{\theta})$. Due to the high ex ante uncertainty about the final allocation, no symmetric efficient equilibrium exists.

Importantly, if the uncertainty concerning bidders' types is substantial, all equilibria of the CCA, which is a dynamic implementation of the VCG auction, are inefficient. The next section shows that no matter how large this uncertainty is, the VCG auction always has efficient equilibria. It is, therefore, the information that is transmitted during the clock phase that may destroy efficiency (even if little information is provided). The result demonstrates that blending two well meant auction design principles (the second-price principle and an open format) may have unintended consequences.

The main intuition for this result can be developed by combining different arguments that we have previously developed. From Proposition 2, we know that in any efficient equilibrium, some types will expand demand. Because of the relative cap and the large type space, the clock must last long in an efficient equilibrium if one bidder is sufficiently strong. The long duration gives some relatively strong types the possibility of weakening the constraints of the activity rules by expanding demand. Because of the high uncertainty, the highest and the lowest types cannot pool at a threshold price in an efficient equilibrium. Consequently, the clock not ending when low types drop out reveals to strong types that their competitor is strong. The spiteful bid of high types then jumps discretely in any supplementary round that follows a longer duration of the clock. Other types will reduce their clock phase demand in anticipation of this behavior, which - given the activity rules-necessarily leads to inefficiencies.

This argument is developed in more detail with the following notation. Note that if $u(0, \underline{\theta})<u(1, \bar{\theta})$, there exists a type $\underline{\theta}^{\prime}>\underline{\theta}$ such that $u\left(0, \underline{\theta}^{\prime}\right)=u(1, \bar{\theta})$ and that the lowest possible efficient share of all types in $\left[\underline{\theta}, \underline{\theta^{\prime}}\right]$ is 0 . Likewise, there is a type $\bar{\theta}^{\prime}<\bar{\theta}$ such that $u\left(1, \bar{\theta}^{\prime}\right)=u(0, \underline{\theta})$, so that the largest possible efficient share of all types in $\left[\bar{\theta}^{\prime}, \bar{\theta}\right]$ is the full supply.

We first argue that in any efficient equilibrium, when the lowest type $\underline{\theta}$ meets a type above $\bar{\theta}^{\prime}$, the final clock price must be $u(0, \underline{\theta})$, which is the clock price at which the lowest type drops out of the auction under truthful bidding. Because of the high uncertainty, the truthful demand of types above $\bar{\theta}^{\prime}$ is the full supply at this price. If the final clock price $p$ was smaller for such a type profile, then at least one of the bidders would have reduced demand, as for these prices $u\left(1, \theta^{\prime}\right)>u(0, \underline{\theta})>p$ for all types $\theta^{\prime}>\bar{\theta}^{\prime}$, so that if none of them would have reduced demand, then aggregate demand would be larger than supply. Given the restrictions imposed by the relative cap, these bidders could not bid marginal utilities on all possibly efficient shares in the supplementary round (Proposition 2). The final clock price for such a type combination cannot be larger than $u(0, \underline{\theta})$ either, as this would imply that $\underline{\theta}$ and some marginally larger types have excessively expanded demand. Demand expansion at these high prices and the requirement that supplementary bids must be at least as high as clock bids lead to the bidder necessarily winning too much or too little. Thus, the clock must end at $u(0, \underline{\theta})$ for these type profiles.

Second, we show that in any efficient equilibrium, types marginally larger than $\underline{\theta}$ will demand truthfully at clock prices slightly larger than $u(0, \underline{\theta})$. By the same reasoning as in 
the previous paragraph, the clock cannot end later than $u_{j}(0)$ for $\theta_{j}>\underline{\theta}$ through demand expansion. Bidders also cannot reduce demand as the relative cap then prevents them from bidding true marginal values on efficient shares, which is necessary for ex post efficiency. As a result, they must bid truthfully at these prices.

Given this behavior of types just above $\underline{\theta}$, we next argue that similar to the reasoning in Proposition 1, types just below $\bar{\theta}^{\prime}$ find it optimal to maximally expand demand in the clock phase for prices $p<u(0, \underline{\theta})$. The reason is that by maximally expanding demand, the clock cannot end at prices $p<u(0, \underline{\theta})$, while they know that in an efficient equilibrium, the clock will continue for them if the rival is sufficiently strong. Expanding demand in the clock implies they discretely increase their supplementary bid on the full supply if the clock stops at a price larger than $u(0, \underline{\theta})$ compared to the situation where the clock stops at $u(0, \underline{\theta})$ and they find it optimal to do so. In anticipation of this behavior, some types in $\left(\underline{\theta}, \underline{\theta}^{\prime}\right]$ will then find it profitable, however, to reduce their demand at $u(0, \underline{\theta})$, ending the clock prematurely to prevent the rival from further raising their costs. Such behavior is inconsistent with efficiency, however, showing that there is no efficient equilibrium.

\subsection{Inefficient equilibria in the quadratic utility model}

This subsection uses the quadratic utility model to present an example of an inefficient equilibrium that illustrates the kind of equilibria that may exist when efficient equilibria do not exist. Thus, the example underlines that Proposition 3 is not due to a general nonexistence of equilibrium. In our example, the final allocation is almost surely inefficient as bidders win either $0,1 / 2$, or the full supply. For simplicity, we consider a CCA in which bidders are informed about aggregate demand in the final clock round.

Let $\sigma<\bar{\theta}-\underline{\theta} \leq 3 / 2 \cdot \sigma$. We have three threshold prices $\tilde{p}^{1}<\tilde{p}^{2}<\tilde{p}^{3}$, and partition the type space into $\left[\underline{\theta}, \theta^{1}\right),\left[\theta^{1}, \theta^{2}\right)$, and $\left[\theta^{2}, \bar{\theta}\right]$. Call $\theta^{3}=\bar{\theta}$ and $\theta^{0}=\underline{\theta}$. Let $I \in\{1,2,3\}$ be the index for type $\theta_{i} \in\left[\theta^{I-1}, \theta^{I}\right.$ ), where the interval is closed for $I=3$. Define $J$ analogously for a bidder with type $\theta_{j}$.

We choose the prices such that

$$
U\left(1 / 2, \theta^{I-1}\right)-\tilde{p}^{I} / 2=0
$$

and choose the cutoff types so that

$$
U\left(1 / 2, \theta^{I}\right)-\tilde{p}^{I} / 2=U\left(1, \theta^{I}\right)-\tilde{p}^{I} .
$$

Thus, at price $\tilde{p}^{I}$, type $\theta^{I-1}$ is indifferent between dropping demand to 0 and bidding for half of the supply, while type $\theta^{I}$ is indifferent between bidding for half of the supply and the full supply. For $\bar{\theta}$, the equality can be an inequality so that the left-hand side is larger than the right-hand side. When bidders have quadratic utility functions, we have $\tilde{p}^{I}=\underline{\theta}-\sigma / 4+(I-1) \cdot \sigma / 2$ and $\theta^{I}=\underline{\theta}+I \cdot \sigma / 2$. 
Strategies In the clock phase, type $\theta_{i} \in\left[\theta^{I-1}, \theta^{I}\right)$ follows the clock demand function

$$
x_{i}(p)= \begin{cases}1 & \text { for } p<\tilde{p}^{I} \\ 0 & \text { for } p \geq \tilde{p}^{I} .\end{cases}
$$

Hence, the clock ends with both bidders demanding 0 if $I=J$, and with one demanding the full supply and the other demanding 0 if $I \neq J$.

The supplementary bids depend on aggregate demand in the final clock round. If the clock ends with aggregate demand of 0 at the final clock price $\tilde{p}$, then $\theta_{i}$ bids

$$
S_{i}^{\tilde{p}^{I}}\left(x \mid x_{i}(\tilde{p})+x_{j}(\tilde{p})=0\right)= \begin{cases}\tilde{p} / 2 & \text { for } x=1 / 2 \\ \tilde{p} & \text { for } x=1 \\ 0 & \text { else. }\end{cases}
$$

If aggregate demand in the last clock round is positive, then the supplementary bidding function is given by

$$
S_{i}^{\tilde{p}^{I}}\left(x \mid x_{i}(\tilde{p})+x_{j}(\tilde{p})>0\right)= \begin{cases}\tilde{p} & \text { for } x=1 \\ 0 & \text { else. }\end{cases}
$$

The supplementary bidding functions clearly satisfy the constraints of the activity rules, and the final cap and the relative cap are binding for strictly positive bids. The difference between the two bidding functions is that in the former case, a positive bid on $1 / 2$ is submitted, whereas in the latter case, no such bid is made.

No incentive to deviate If bidders belong to the same partition group, i.e., $I=J$, then both win half of the supply at the CCA price $\tilde{p}^{I} / 2$. The construction of $\tilde{p}^{I}$ and the cutoff types make it clear that these bidders prefer this outcome over winning the full supply at a price of $\tilde{p}^{I}$. Bidders could win $x>1 / 2$ by deviating to demanding $x$ at the final clock price $\tilde{p}^{I}$ (instead of 0 ). The CCA price for $x$ would then be $\tilde{p}^{I}$. It is clear that this gives less surplus than the full supply, which in turn is a worse outcome than winning $1 / 2$. Bidder $i$ could also win a share $0<x<1 / 2$ at the CCA price of $\tilde{p}^{I} / 2$ by deviating in the supplementary phase that follows $\tilde{p}^{I}$ and zero aggregate demand. Again, this leads to a lower surplus than winning $1 / 2$.

Next consider the case where $\theta_{i}$ and $\theta_{j}$ are such that $I<J$. The clock ends at $\tilde{p}^{I}$ with market clearing. Bidder $j$ wins the full supply at the CCA price $\tilde{p}^{I}$. The construction of the prices and cutoff types is such that the stronger bidder prefers winning 1 at CCA price $\tilde{p}^{I}$ over $1 / 2$ at the CCA price $\tilde{p}^{I} / 2$. The lowest price at which bidder $i$ could win a positive amount is $\tilde{p}^{J}$, which is so high that bidder $i$ would incur a loss. Hence, bidders do not have an incentive to deviate, even if they know the competitor's type. Hence, the proposed strategies form an ex post equilibrium. There are also no profitable deviations in the spite dimension of the preferences. If a bidder demanded $x>0$ at $\tilde{p}^{I}$, then he would lose the possibility of winning $1 / 2$ at a price at which he makes a positive surplus. Hence, a bidder cannot further raise his rival's costs without decreasing his own expected surplus. 
Discussion The inefficiency in this equilibrium can be quite substantial. For example, if a type marginally below the cutoff meets a type marginally above the cutoff, the efficient allocation has both bidders approximately winning half the supply. The equilibrium outcome is, however, one where the slightly stronger type wins the full supply. For $\theta^{1}$ with $\sigma=\underline{\theta}$, this amounts to a welfare loss of $20 \%$.

If the uncertainty is larger (or smaller) than assumed in this subsection, then it is easy to extend the equilibrium construction to more than three threshold prices.

\section{The VCG MECHANISM}

To better understand the implications of having a clock phase for raising the rival's cost, we now briefly analyze the VCG mechanism. The purpose of this section is twofold. First, we want to establish how we see the weak preference for raising the rival's cost as an alternative way to select among the many equilibria of the VCG. Second, we want to show that, independent of the size of the uncertainty, the VCG always has efficient equilibria when bidders are spiteful. The contrast with the result from the CCA of the previous section reinforces the point that it is the dynamic element of the CCA, i.e., the clock phase, that is responsible for the inefficiency result via an effect that is similar to the ratchet effect.

We first show that under standard preferences, the outcome of applying iterative elimination of weakly dominated strategies (IEDS) to the VCG mechanism is always efficient, but that the payments are undetermined and depend on the way IEDS is implemented. Truthful bidding is one of the strategies that survives IEDS, but, depending on the order of elimination, other strategies may survive IEDS as well. Bidders have to bid true marginal values on possible efficient shares in the interval $\left[\underline{x}_{i}, \bar{x}_{i}\right]$ so as to get the efficient share. Outside the interval $\left[\underline{x}_{i}, \bar{x}_{i}\right]$, bidders may bid differently as, depending on the order of elimination, bids on these shares may not be pivotal. As for weaker bidders, it is always the case that $\bar{x}_{i}<1$, these bidders have a range of shares for which the bid is undetermined by IEDS, and the choice of these bids determines how much competitors have to pay. Accordingly, the payments in the VCG mechanism may well differ from the payments under truthful bidding.

Proposition 4. In the VCG mechanism with standard preferences, any strategy profile that survives any process of IEDS implements the efficient allocation. The VCG payments depend, however, on the order in which weakly dominated strategies are eliminated and on the choice of strategy profile that survives IEDS.

One way to resolve the indeterminacy related to payments is to impose that bidders play their weakly dominant strategy. The lexicographic spiteful preferences may be viewed as a more plausible alternative.

Under spiteful preferences, truthful bidding is not an equilibrium in the VCG mechanism. To see this, suppose other bidders bid truthfully and consider a weak enough bidder with type $\theta_{i}$ for whom $\bar{x}_{i}<1$. Without lexicographic preferences, bidder $i$ is indifferent between some bids on $\left(\bar{x}_{i}, 1\right]$. A lexicographic bidder knows, however, that he 
can increase the price other bidders have to pay. The easiest way to do so is to increase the bid $S_{i}(1)$ on the full supply as much as possible under the constraint that it is not winning. ${ }^{18}$ He never wins the full supply in an efficient equilibrium if for all $\theta_{j} \in[\underline{\theta}, \bar{\theta}]$,

$$
S_{i}(1) \leq S_{i}\left(x_{i}^{*}\left(\theta_{i}, \theta_{j}\right)\right)+S_{j}\left(x_{j}^{*}\left(\theta_{j}, \theta_{i}\right)\right) .
$$

The right-hand side of (3) depends on the type of the other bidder and is minimized if the other bidder has the lowest possible type $\underline{\theta}$. Hence, given our formulation of the spite motive, bidder $i$ wants to set the bid on 1 equal to the minimal value of the efficient allocation given bidder $i$ 's type. If both bid true utility on $\left[\underline{x}_{i}, \bar{x}_{i}\right]$, then the optimal bid is $S_{i}(1)=V_{i}(\underline{\theta})$. Thus, both bidders can use their private information and their knowledge about the lowest possible type of their rival to raise the bid on the full supply. Types that can win everything in an efficient equilibrium maximize the rival's payment by bidding truthfully, in which case $S_{i}(1)=U_{i}(1)=V_{i}(\underline{\theta})$.

The next proposition determines an efficient equilibrium under lexicographic preferences where bidders bid truthfully on all possible shares, apart from 1 if the type is low enough. ${ }^{19}$ The equilibrium strategies are increasing in $x$, but not necessarily continuous at 1.

Proposition 5. Let bidders have lexicographic spiteful preferences. The strategy profile in which bidder $i=1,2$ with type $\theta_{i}$ plays

$$
S_{i}(x)= \begin{cases}U_{i}(x) & \text { for } 0 \leq x<1 \\ V_{i}(\underline{\theta}) & \text { for } x=1\end{cases}
$$

forms an equilibrium of the VCG auction. Under standard preferences, there is a process of IEDS such that this strategy profile is iteratively undominated.

In strategy profile (4), all bidders bid true utility on all shares smaller than 1 . No bidder can further raise the VCG price without running the risk of winning, as the other bidder's type may be such that the value of the efficient allocation is minimal. Hence, the strategy profile is an equilibrium under lexicographic preferences for raising the rival's costs. Note that the strategy profile in (4) implements the efficient allocation and survives the IEDS of the proof of Proposition 4.

It is also important to note that all types $\theta_{i}>\underline{\theta}$ make positive surplus. This is because bidders do not want to risk winning the full supply and, therefore, are restricted in raising their rival's cost by the lowest possible efficient value $V_{i}(\underline{\theta})$. If bidders knew their rival's type, they would fully expropriate them in any equilibrium where bidders bid valuation on the possibly efficient shares $\left[\underline{x}_{i}, \bar{x}_{i}\right]$. Thus, in the VCG mechanism, bidders benefit from rivals being uncertain about their type.

\footnotetext{
${ }^{18} \mathrm{He}$ could also increase his bid on other $x \in\left(\bar{x}_{i}, 1\right)$, but this does not create any benefit.

${ }^{19}$ This is not the only equilibrium when bidders have lexicographic preferences. It is clear that bidders never want to bid above value on possible efficient shares. To protect themselves against others raising their price, bidders may, however, reduce their own bids on the domain of possibly efficient allocations without affecting their marginal bids.
} 


\section{DisCUSSION AND CONCLUSION}

This paper provides a full equilibrium analysis of the CCA where the strategic interaction between the clock phase and the supplementary round is studied in an environment where bidders not only care about their own payoff but also (lexicographically) about how much rivals pay. We have two main results. First, there does not exist an efficient equilibrium of the CCA that fully reveals the type of the competitor in the clock phase. Our second main result is that the CCA is inefficient if the uncertainty concerning final allocations is relatively large.

It is difficult to assess whether real-world CCAs have been efficient, as this would require knowing bidders' utility functions. However, many of the equilibrium features of the CCAs we have highlighted show similarities to observed features of CCAs. Without pretending that there are no alternative explanations for these phenomena, we provide the following observations. First, after the 2013 auction, the Austrian regulator RTR observed that during a large part of the clock phase, bidders' demanded close to their full spectrum caps. This is in line with our examples on clock-pooling and clock-semiseparating equilibria, and is explained by our result on demand expansion in the clock phase. Second, the Austrian mobile network operator Telekom Austria (2013) indicates in a press release after the auction that the clock phase ended with excess supply in key spectrum bands. According to the Austrian regulator RTR, this did not prevent the bidders from bidding aggressively in the supplementary round. ${ }^{20}$ This is also in line with our examples on clock-pooling and clock-semi-separating equilibria, where we argue that bidders create excess supply purposefully to obfuscate their type to prevent rivals from raising their costs. ${ }^{21}$

Ausubel and Baranov (2014) suggest alternative activity rules with the purpose of providing bidders with stricter incentives to bid according to their intrinsic preferences. They propose replacing the relative cap we use in this paper by GARP (the generalized axiom of revealed preference). We observe that bidders do not violate GARP in any of our equilibria. We conclude, therefore, that most of our results continue to hold if we were to adopt the GARP activity rule.

We end our paper by briefly discussing the robustness of our results to changes in the model. First, many of our findings hold when there are more than two bidders. Equilibria with more than two bidders feature coordination on how the rivals' costs are raised. In equilibrium, this coordination is not an issue, but characterizing the equilibrium is more difficult. Restricting the analysis to two bidders also has a certain virtue beyond simplicity, as all relevant information may be revealed in a fully revealing equilibrium. When two bidders bid truthfully in the clock phase, the final clock price reveals the other bidder's type. With more than two bidders, however, one learns at most only the "sum of types." The two bidder case, therefore, allows the clearest test of the CCA. Second, it might be that values are not private, but interdependent. In this case, information revelation in the clock may improve efficiency. While this may be true, the underlying

\footnotetext{
${ }^{20}$ See https://www.rtr.at/en/pr/PI28102013TK.

${ }^{21}$ The clock phase of the Canadian $700 \mathrm{MHz}$ auction also ended with excess supply even though these units were allocated in the supplementary round.
} 
economic forces described in this paper still hold. Low types know that they cannot win the full supply, so they expand demand so as to place high bids on the full supply to raise rivals' costs. Bidders are, therefore, still reluctant to reveal their private information because they know that by doing so they will be exploited. Thus, the trade-off between information revelation and efficiency we have uncovered in this paper is likely to remain important in more complicated settings.

\section{Appendix: Omitted PROOFS}

Proposition 1. There does not exist an efficient clock-separating equilibrium in the CCA.

Proof. Suppose an efficient clock-separating equilibrium exists. Clock separation requires that demand is monotone in type, i.e., $\theta_{i} \geq \theta_{j}$ implies $x_{i}(p) \geq x_{j}(p)$. Let $T$ be an open neighborhood of the type profile $\underline{\theta} \in[\underline{\theta}, \bar{\theta}]^{2}$ so that for any $\theta \in T$, all bidders are winners in the efficient allocation. The equilibrium strategy profile must have the following properties.

First, the clock must end with market clearing almost surely. Suppose there is a positive probability, i.e., an open set of type profiles $T^{\prime} \subseteq T$, that the clock ends with excess supply. The clock ends with excess supply only if a bidder uses a demand function with discrete downward jumps. Without loss of generality, let bidder 1 make a jump that ends the clock for type profile $\theta \in T^{\prime}$ at $\tau(\theta)=p$. Consider type $\theta_{2}^{\prime}$ being slightly smaller than $\theta_{2}$. Then the clock must end with excess supply at $\tau\left(\theta_{1}, \theta_{2}^{\prime}\right)$ by bidder 2 making downward jumps, because $\tau$ is increasing in $\theta_{2}$. Fix $\theta_{2}^{\prime}$ slightly smaller than $\theta_{2}$ such that $\tau\left(\theta_{1}, \theta_{2}^{\prime}\right)=p^{\prime}<p$ and bidder 1's demand with type $\theta_{1}$ has no jumps on $\left[p^{\prime}, p\right)$. Note that for a given $\theta_{j}$, the function $\tau\left(\theta_{i}, \theta_{j}\right)$ is strictly increasing in $\theta_{i}$ and, therefore, continuous almost everywhere. Hence, there exists a type $\theta_{1}^{\prime}$ slightly larger than $\theta_{1}$ such that $\tau\left(\theta_{1}^{\prime}, \theta_{2}^{\prime}\right)=p^{\prime \prime}$ and $p^{\prime}<p^{\prime \prime}<p$. At $p^{\prime \prime}$, it is bidder 1's discrete decrease that ends the clock. Since demand functions are monotone in type, it must be that type $\theta_{1}$ drops demand at $p^{\prime \prime}$, a contradiction.

Second, the relative cap must be binding for relevant shares in $\left(x_{i}^{*}, 1\right]$ in any supplementary phase on the equilibrium path. Since the clock ends with market clearing almost surely and the equilibrium is efficient, demand in the final clock round must be the respective efficient shares. This follows from the definition of the final cap rule. In addition, the clock ending with market clearing and the final cap imply that the supplementary bids determine only the other bidder's CCA price. If the relative cap was not binding, then a spiteful bidder could further raise the supplementary bids on shares that determine other bidders' CCA prices relative to the efficient share without changing the final allocation. The proposed equilibrium strategy would then not be a best response in the spite dimension.

Third, bidders need to demand truthfully for $p \in\left[u_{i}\left(\bar{x}_{i}\right), u_{i}\left(\underline{x}_{i}\right)\right]$. The clock ends with market clearing and the relative cap is binding. Bidder $j$ wants to win the efficient share only if $u_{j}\left(x^{*}\right)=s_{i}\left(1-x^{*}\right)=u_{i}\left(1-x^{*}\right)$. Hence, bidder $i$ must demand truthfully. 
We now show that, given these properties, there is a profitable deviation from the clock-separating equilibrium strategy. This deviation leads to the same expected utility in the first dimension of the preferences, but to strictly higher CCA prices for some possible final clock prices. Bidder $i$ deviates by first expanding demand for some prices strictly above $u_{i}\left(\bar{x}_{i}\right)$, and bids truthfully at some price $p>u_{i}\left(\bar{x}_{i}\right)$ and from then on. If the clock ends at $p$, it almost surely ends with excess supply. Other bidders do not see the deviation and believe that the clock ended with market clearing. They fully raise the supplementary bids to $s_{j}(x)=u_{j}(x)$ for $x \in\left[x_{j}(p), \bar{x}_{j}\right]$. Hence, a suitable level of $S_{i}\left(x_{i}(p)\right)$ and true marginal values $s_{i}(x)=u_{i}(x)$ on $\left[x_{i}(p), \bar{x}_{i}\right]$ implement the efficient allocation. The CCA price for the deviating bidder is the same as under the initial strategy, since the CCA price is independent of the final clock price. The CCA price for the other bidder is not less than the "equilibrium" price if the clock ends at $p$. If the clock does not end at $p$, it will end at a higher clock price with market clearing. The deviation weakened the constraints of the activity rule; hence, the spiteful bids in $\left(\bar{x}_{i}, 1\right]$ are strictly larger than those of the initial strategy and lead to a higher CCA price for the other bidder.

Proposition 3. Let $u(1, \bar{\theta})>u(0, \underline{\theta})$. Due to the high ex ante uncertainty about the final allocation, no symmetric efficient equilibrium exists.

Proof. The following lemma describes the necessary equilibrium clock behavior. The remainder of the proof concerns the supplementary bids on the equilibrium path. Let $\tilde{x}_{i}$ denote the truthful demand of $\theta_{i}$ at $\bar{p}$.

Lemma 1. Let $u(0, \underline{\theta})<u(1, \bar{\theta})$. In any symmetric efficient equilibrium, there is an $\epsilon>0$ such that type $\theta_{i} \in\left[\overline{\bar{\theta}}^{\prime}-\epsilon, \bar{\theta}^{\prime}\right)$ expands demand for some prices $p<\bar{p}=u(0, \underline{\theta})$. Moreover, there exists a $\delta>0$ such that all types in $\left[\underline{\theta}, \underline{\theta^{\prime}}\right] \cup\left[\bar{\theta}^{\prime}-\epsilon, \bar{\theta}\right]$ demand truthfully at $p \in[\bar{p}$, $\bar{p}+\delta]$.

Proof. We first prove three auxiliary claims and then the lemma's claims in the order that they appear. We begin by showing that whenever a relatively weak type demands a possibly efficient share in the clock phase on the equilibrium path, this must be the truthful demand.

Claim 1. Let $u(0, \underline{\theta})<u(1, \bar{\theta})$ and $\theta_{j} \in\left[\underline{\theta}, \underline{\theta^{\prime}}\right]$. In any efficient equilibrium and for all $p \leq \tau\left(\bar{\theta}, \theta_{j}\right)$, if $x_{j}(p) \in\left(0, \bar{x}_{j}\right]$, then $u_{j}\left(x_{j}(p)\right)=p$, and if $x_{j}(p)=0$, then $u_{j}(0) \leq p$.

Proof. Let $u(0, \underline{\theta})<u(1, \bar{\theta})$ and consider any efficient equilibrium. Let $\theta_{j} \in\left[\underline{\theta}, \underline{\theta^{\prime}}\right]$ demand $x_{j}(p) \in\left[0, \bar{x}_{j}\right]$. The proof of Proposition 2 shows that bidder $\theta_{j}$ cannot reduce demand, as this would prevent him from bidding true marginal values in any subsequent supplementary phase. Hence, $u_{j}\left(x_{j}(p)\right) \leq p$.

To prove that there cannot be demand expansion, we first show a property any efficient equilibrium must exhibit. Consider any efficient equilibrium and define the equilibrium price type $\theta_{j}$ has to pay when facing $\theta_{i}$ as

$$
p_{i}^{\mathrm{CCA}}\left(\theta_{j}\right)=\max _{y} S_{i}^{\tau\left(\theta_{i}, \theta_{j}\right)}(y)-S_{i}^{\tau\left(\theta_{i}, \theta_{j}\right)}\left(x_{i}^{*}\left(\theta_{j}\right)\right) .
$$


Depict this function as $p_{i}^{\mathrm{CCA}}\left(\theta_{j}\right)=\xi\left(\theta_{i}, \theta_{j}\right)-U_{i}\left(x_{i}^{*}\left(\theta_{j}\right)\right)$. We first show that $\xi$ is independent of $\theta_{j}$ so that bidder $j$ 's type enters the CCA price only via the efficient share. It is clear that $p_{i}^{\mathrm{CCA}}$ cannot have any jumps, as this would lead to discretely different prices for marginally different types. In an efficient ex post equilibrium, we must have

$$
\theta_{j} \in \arg \max _{\tilde{\theta}_{j}} U_{j}\left(x^{*}\left(\tilde{\theta}_{j}, \theta_{i}\right)\right)-p_{i}^{\mathrm{CCA}}\left(\tilde{\theta}_{j}\right)
$$

i.e., no type has an incentive to pretend to be a different type. Consider the first derivative of the maximization problem with respect to $\tilde{\theta}_{j}$,

$$
\frac{\partial x_{i}^{*}\left(\tilde{\theta}_{j}\right)}{\partial \tilde{\theta}_{j}}\left[u_{i}\left(x_{i}^{*}\left(\tilde{\theta}_{j}\right)\right)-u_{j}\left(1-x_{i}^{*}\left(\tilde{\theta}_{j}\right)\right)\right]-\frac{\partial \xi\left(\theta_{i}, \tilde{\theta}_{j}\right)}{\partial \tilde{\theta}_{j}},
$$

and impose the optimality of truthful reporting, i.e.,

$$
\left.\frac{\partial x_{i}^{*}\left(\tilde{\theta}_{j}\right)}{\partial \tilde{\theta}_{j}}\left[u_{i}\left(x_{i}^{*}\left(\tilde{\theta}_{j}\right)\right)-u_{j}\left(1-x_{i}^{*}\left(\tilde{\theta}_{j}\right)\right)\right]\right|_{\tilde{\theta}_{j}=\theta_{j}}-\frac{\partial \xi\left(\theta_{i}, \tilde{\theta}_{j}\right)}{\partial \tilde{\theta}_{j}}=0 .
$$

The first term equals 0 , as either the efficient share is in the interior so that the marginal utilities are equal or it is on the boundary so that the efficient share does not depend locally on $\theta_{j}$. Hence, in an efficient equilibrium, $\xi$ cannot depend on $\theta_{j}$ and we have

$$
p_{i}^{\mathrm{CCA}}\left(\theta_{j}\right)=\xi_{i}-U_{i}\left(x_{i}^{*}\left(\theta_{j}\right)\right) .
$$

Coming back to the proof of the claim, suppose that $p \leq \tau\left(\bar{\theta}, \theta_{j}\right)$ and $\hat{x}=x_{j}(p)$ with $u_{j}(\hat{x})<p$. When the clock ends at $\tau\left(\bar{\theta}, \theta_{j}\right)$, type $\theta_{j}$ 's highest supplementary bid is $\max _{y} S_{j}(y)=\xi_{j}$, as $S_{j}(0)=U_{j}\left(x^{*}\left(\theta_{j}, \bar{\theta}\right)\right)=0$. The CCA price for share $\hat{x}$ when the clock ends at $\tau\left(\bar{\theta}, \theta_{j}\right)$ is, therefore,

$$
\xi_{j}-S_{j}^{\tau\left(\bar{\theta}, \theta_{j}\right)}(\hat{x}) \leq \xi_{j}-p \hat{x}<\xi_{j}-U_{j}(\hat{x}),
$$

where the first inequality follows from clock bids remaining valid (i.e., the activity rules' constraint from below), while the second inequality follows from demand expansion. Note that there is a type $\hat{\theta}$ such that $\hat{x}=x^{*}\left(\theta_{j}, \hat{\theta}\right)$. This type gets $1-\hat{x}$ at a price that is smaller than (5). This is a contradiction.

Claim 2. Let $u(0, \underline{\theta})<u(1, \bar{\theta})$. In any symmetric efficient equilibrium, for all $\theta_{j} \in\left[\underline{\theta}, \underline{\theta}^{\prime}\right]$, we have $\tau\left(\bar{\theta}, \theta_{j}\right) \leq u_{j}(0)$.

Proof. Suppose to the contrary that there is a symmetric and efficient equilibrium and a type $\theta_{j} \in\left[\underline{\theta}, \underline{\theta}^{\prime}\right]$ such that $\hat{p}=\tau\left(\bar{\theta}, \theta_{j}\right)>u_{j}(0)$. Claim 1 implies that whenever bidder $j$ demands a possibly efficient share, bidder $j$ must demand truthfully. The clock ends, however, at $u_{j}(0)$ at the latest under truthful bidding. Hence, $\tau\left(\bar{\theta}, \theta_{j}\right)>u_{j}(0)$ can be true only if bidder $j$ demands $x_{j}(p)>\bar{x}_{j}$ for $p<\hat{p}$ and 0 at $\hat{p}$. In a type-symmetric equilibrium, the clock necessarily ends for at least all $\theta_{i} \geq \theta_{j}$ at $\hat{p}$. In the supplementary phase that follows the clock ending at $\hat{p}$, efficiency requires $\theta_{j}$ bidding true marginal 
utility on [0,1/2]. As $S^{\hat{p}}(0)=0$, this implies bidding true utility, i.e., $S_{j}^{\hat{p}}(x)=U_{j}(x)$ for $x \in[0,1 / 2]$.

We now show that the efficient allocation $(1 / 2,1 / 2)$ is not implemented if both bidders have type $\theta_{j}$. Let $\delta>0$ be small enough so that $\hat{p}-\delta>u_{j}(0)$ and let $x=x_{j}(\hat{p}-\delta)>$ $1 / 2$. For the equilibrium to be efficient, we must have

$$
2 S_{j}^{\hat{p}}(1 / 2)=2 U_{j}(1 / 2) \geq S_{j}^{\hat{p}}(x)+S_{j}^{\hat{p}}(1-x) \geq(\hat{p}-\delta) x+U_{j}(1-x),
$$

where we use truthful bidding on possible efficient shares and the fact that clock bids remain valid. The inequality simplifies to

$$
\begin{aligned}
& U_{j}(1 / 2)+\int_{1-x}^{1 / 2} u_{j}(y) d y \geq(\hat{p}-\delta) x \\
& \quad \Rightarrow \quad u_{j}(0) / 2+u_{j}(1-x)(x-1 / 2)>(\hat{p}-\delta)(1 / 2+x-1 / 2) .
\end{aligned}
$$

The inequality is false, however, as $\hat{p}-\delta>u_{j}(0) \geq u_{j}(1-x)$.

Claim 3. Let $u(0, \underline{\theta})<u(1, \bar{\theta})$. In any symmetric efficient equilibrium, there is an $\epsilon>0$ such that for all $\theta_{i} \in\left[\bar{\theta}^{\prime}-\epsilon, \bar{\theta}\right]$, we have $\tau\left(\theta_{i}, \underline{\theta}\right)=\bar{p}=u(0, \underline{\theta})$.

Proof. Suppose to the contrary that there is a symmetric efficient equilibrium such that for all $\epsilon>0$, there is a type $\theta_{i} \in\left[\bar{\theta}^{\prime}-\epsilon, \bar{\theta}\right]$ with $\tau\left(\theta_{i}, \underline{\theta}\right) \neq u(0, \underline{\theta})$. There are three exhaustive cases to consider. First, Claim 2 shows that $\tau\left(\theta_{i}, \underline{\theta}\right)>u(0, \underline{\theta})$ cannot occur for any $\theta_{i}$ in a symmetric efficient equilibrium, because if it happened for $\theta_{i}$, it would also happen for $\bar{\theta}$ in a monotone equilibrium. Second, we show that it is impossible that $\tau\left(\theta_{i}, \underline{\theta}\right)<u(0, \underline{\theta})$ for any $\theta_{i} \in\left[\bar{\theta}^{\prime}, \bar{\theta}\right]$. The third case considers types just below $\bar{\theta}^{\prime}$.

It is straightforward to see that in any efficient equilibrium it cannot be that for type $\theta_{i} \in\left[\bar{\theta}^{\prime}, \bar{\theta}\right]$ we have $\tau\left(\theta_{i}, \underline{\theta}\right)=p<u(0, \underline{\theta})$. Proposition 2 tells us that in an efficient equilibrium bidders do not reduce demand at this price. If $p<u(0, \underline{\theta}) \leq u(1, \bar{\theta})$, the truthful demand of types just below $\bar{\theta}^{\prime}$ at $p$ equals the full supply, while the truthful demand of the low type is strictly positive. Hence, the clock cannot end at $\tau\left(\theta_{i}, \underline{\theta}\right)=p<u(0, \underline{\theta})$.

We now turn our attention to types "just below" $\bar{\theta}^{\prime}$, as it could be that for every $\epsilon>0$ there is a type $\theta_{i} \in\left(\bar{\theta}^{\prime}-\epsilon, \bar{\theta}^{\prime}\right)$ with $\tau\left(\theta_{i}, \underline{\theta}\right)<u(0, \underline{\theta})$. From the previous case it follows that $\tau\left(\theta_{i}, \underline{\theta}\right)$ must be increasing in $\theta_{i}$, because otherwise there would be types just below $\bar{\theta}^{\prime}$ whose truthful demand is the full supply and who reduce demand, contradicting Proposition 2. We will show that for types just below $\bar{\theta}^{\prime}$, the clock must end at $u(0, \underline{\theta})$ if they meet the lowest type. It cannot end earlier due to an argument that relies on bidders having spiteful lexicographic preferences and which is akin to the proof of Proposition 1. For clock prices just below $u(0, \underline{\theta})$, the truthful demand of types just below $\bar{\theta}^{\prime}$ is almost the full supply, while low types' truthful demand is slightly above 0 . The function $\tau\left(\theta_{i}, \underline{\theta}\right)$ increases in $\theta_{i}$ if demand is decreased continuously. Efficiency of equilibrium and the final cap require bidders to lower demand truthfully. Hence, the lowest type is active for all $p<u(0, \underline{\theta})$. The marginal supplementary bids when the clock ends at $u(0, \underline{\theta})$ must satisfy the relative cap $s(x, \underline{\theta}) \leq u(x, \underline{\theta})$, which follows from truthful bidding at prices just below clock price $u(0, \underline{\theta})$. Thus, the lowest type bids true utility on shares (slightly) 
above 0 . This follows from the relative cap being necessarily binding in an efficient and information revealing equilibrium. As a result, types just below $\bar{\theta}^{\prime}$ have no incentive to lower demand truthfully at prices just below $u(0, \underline{\theta})$, because they can expand demand until $u(0, \underline{\theta})$ and still get the efficient share at the same CCA price if they meet a very low type.

The first claim of the lemma follows directly from the previous claim. For types just below $\bar{\theta}^{\prime}$, the clock does not end before $u(0, \underline{\theta})$ in an efficient equilibrium. Thus, these types will expand demand for clock prices $p<u(0, \underline{\theta})=\bar{p}$ in equilibrium. The demand expansion weakens the (necessarily) binding constraint of the relative cap.

The second claim of the lemma is that there is a $\delta>0$ such that types in $\left[\underline{\theta}, \underline{\theta^{\prime}}\right] \cup$ $\left[\bar{\theta}^{\prime}-\epsilon, \bar{\theta}\right]$ demand truthfully for $p \in[\bar{p}, \bar{p}+\delta]$. Bidders cannot reduce demand due to Proposition 2. The proof of Claim 2 rules out $\theta_{j} \in\left[\underline{\theta}, \underline{\theta}^{\prime}\right]$ demanding $x>\bar{x}_{j}$ for $p<u_{j}(0)$ and $x_{j}\left(u_{j}(0)\right)=0$. Claim 1 then shows that $\theta_{j} \in\left[\underline{\theta}, \underline{\theta}^{\prime}\right]$ bids truthfully for clock price $p \in[\bar{p}, \bar{p}+\delta]$. Type $\theta_{i} \in\left[\bar{\theta}^{\prime}-\epsilon, \bar{\theta}\right]$ also has to bid truthfully, as the low types bid truthfully. Demand expansion of a type whose truthful demand is arbitrarily close to the full supply at $p \in[\bar{p}, \bar{p}+\delta]$ goes along with the possibility of market clearing. As the equilibrium is efficient, bidders have to demand truthfully at prices at which the clock can end with market clearing.

We prove the proposition by showing that types just below $\bar{\theta}^{\prime}$ can make the CCA price dependent on the final clock price and this incentivizes some weak bidders to pool with lower types by reducing demand. Consider type $\theta_{i}$ just below $\bar{\theta}^{\prime}$ that expands demand for prices just below $\bar{p}$. Claim 3 of the proof of the lemma shows that the lowest equilibrium final clock price of $\theta_{i}$ is $\bar{p}$. Let $\theta_{j}$ denote the highest type for which the clock may end at $\bar{p}$ when meeting $\theta_{i}$ under truthful bidding, that is, $u_{i}\left(\tilde{x}_{i}\right)=u_{j}\left(\tilde{x}_{j}\right)$. It is clear that $\theta_{j}>\underline{\theta}$.

When the clock ends at $\bar{p}$, efficiency requires the lowest type to bid true utility in a neighborhood of 0 in the supplementary phase. This follows from $S^{\bar{p}}(0)=0$, the necessity of bidding true marginal values on possible efficient shares, and the clock ending at $\bar{p}$ for all types in $\left(\bar{\theta}^{\prime}-\epsilon, \bar{\theta}\right]$. Hence, when the clock stops at $\bar{p}$, bidder $i$ 's supplementary bid on the full supply must be

$$
S_{i}^{\bar{p}}(1)=\min \left\{S_{i}^{\bar{p}}\left(\tilde{x}_{i}\right)+\bar{p}\left(1-\tilde{x}_{i}\right), S_{i}^{\bar{p}}\left(\bar{x}_{i}\right)+\underline{U}\left(1-\bar{x}_{i}\right)\right\} .
$$

Note that $\tilde{x}_{i}=x^{*}\left(\theta_{i}, \theta_{j}\right)<\bar{x}_{i}=x^{*}\left(\theta_{i}, \underline{\theta}\right)$ for types just below $\bar{\theta}^{\prime}$. Since the clock ends at $\bar{p}$ when bidder $i$ meets a type below $\theta_{j}$, bidder $i$ must bid true marginal values on $\left[\tilde{x}_{i}, \bar{x}_{i}\right]$ in the supplementary phase when the clock ends at $\bar{p}$ in an efficient equilibrium, i.e., $S_{i}^{\bar{p}}\left(\bar{x}_{i}\right)=S_{i}^{\bar{p}}\left(\tilde{x}_{i}\right)+U_{i}\left(\bar{x}_{i}\right)-U_{i}\left(\tilde{x}_{i}\right)$.

Now we show that the relative cap is slack for $\theta_{i}$ in the supplementary phase if the clock ends at $\bar{p}$, that is, $S_{i}^{\bar{p}}(1)=S_{i}^{\bar{p}}\left(\bar{x}_{i}\right)+\underline{U}\left(1-\bar{x}_{i}\right)<S_{i}^{\bar{p}}\left(\tilde{x}_{i}\right)+\bar{p}\left(1-\tilde{x}_{i}\right)$. Suppose the inequality was false. Inserting the expression for $S_{i}^{\bar{p}}\left(\bar{x}_{i}\right)$ and simplifying yields

$$
\begin{aligned}
& \bar{p}\left(1-\tilde{x}_{i}+\bar{x}_{i}-\bar{x}_{i}\right) \leq U_{i}\left(\bar{x}_{i}\right)-U_{i}\left(\tilde{x}_{i}\right)+\underline{U}\left(1-\bar{x}_{i}\right) \\
& \quad \Rightarrow \quad \bar{p}\left(1-\bar{x}_{i}\right)+\bar{p}\left(\bar{x}_{i}-\tilde{x}_{i}\right)<u_{i}\left(\tilde{x}_{i}\right)\left(\bar{x}_{i}-\tilde{x}_{i}\right)+\bar{p}\left(1-\bar{x}_{i}\right) .
\end{aligned}
$$


The implication uses decreasing marginal values (e.g., $u(0, \underline{\theta}) \cdot x>\underline{U}(x))$ and $\bar{p}=u(0, \underline{\theta})$. Hence, the last inequality is false and the relative cap must be slack in the supplementary phase that follows $\bar{p}$. The presence of types close to $\underline{\theta}$ therefore limits the types just below $\bar{\theta}^{\prime}$ from fully raising the supplementary bid on the entire supply after the clock ends at $\bar{p}$.

As the clock continues after $\bar{p}$, types just below $\bar{\theta}^{\prime}$ learn that the competitor's type is at least $\theta_{j}>\underline{\theta}$. Let $p^{*} \in(\bar{p}, \bar{p}+\delta)$, where the neighborhood is given by Lemma 1 . As demand is lowered truthfully, the clock can end with market clearing at clock price $p^{*}>\bar{p}$ in equilibrium. In the subsequent supplementary phase, bidder $i$ will raise the bid on the full supply so that the relative cap is binding. For shares in $\left[x_{i}\left(p^{*}\right), \tilde{x}_{i}\right]$, the relative cap requires $s_{i}(x) \leq u_{i}(x)$ due to truthful bidding in the clock phase. Bidder $i$ will also take these constraints as binding and will bid $S_{i}\left(\tilde{x}_{i}\right)=S_{i}\left(x_{i}^{*}\right)+U_{i}\left(\tilde{x}_{i}\right)-U_{i}\left(x_{i}^{*}\right)$. As a result, the CCA price for types around $\theta_{j}$ jumps from $U_{i}\left(\bar{x}_{i}\right)+\underline{U}\left(1-\bar{x}_{i}\right)-U_{i}\left(x_{i}^{*}\right)$ to $U_{i}\left(\tilde{x}_{i}\right)+\bar{p}\left(1-\tilde{x}_{i}\right)-U_{i}\left(x_{i}^{*}\right)$. This discrete increase in the CCA price incentivizes types marginally larger than $\theta_{j}$ to inefficiently reduce demand at $\bar{p}$ to avoid the jump in the CCA price. Thus, there is no efficient equilibrium.

Proposition 4. In the VCG mechanism with standard preferences, any strategy profile that survives any process of IEDS implements the efficient allocation. The VCG payments depend, however, on the order in which weakly dominated strategies are eliminated and on the choice of strategy profile that survives IEDS.

Proof. First we show that with standard preferences, any strategy profile that survives any process of IEDS implements the efficient allocation. This proof has three parts. First, we show that bidding truthfully is always an optimal strategy. Therefore, it survives any process of iteratively eliminating weakly dominated strategies. Second, we argue that any bidder must be indifferent between any strategy that survived the IEDS and truthful bidding. In the third and final step, we show that only the efficient allocation can be implemented by strategies that survive IEDS. We use the following notation. The set $\mathcal{S}_{i}$ is the set of strategies that survived IEDS for a bidder with type $\theta_{i}$. The set of iteratively undominated strategy profiles is denoted as $\mathcal{S}=\mathcal{S}_{1} \times \mathcal{S}_{2}$.

First, bidding truthfully is always an optimal strategy, i.e., it is a best response against any strategy profile of the other bidder $S_{j}$. To see this, let the other bidder use $S_{j}$ and let $\hat{\mathbf{x}}$ denote the allocation implemented by the profile $\left(U_{i}, S_{j}\right)$, that is, $U_{i}\left(\hat{x}_{i}\right)+S_{j}\left(\hat{x}_{j}\right) \geq$ $U_{i}\left(x_{i}\right)+S_{j}\left(x_{j}\right)$ for all other feasible allocations $\mathbf{x}$. This inequality also says that the surplus of bidder $i$ is at least as large under the allocation $\hat{\mathbf{x}}$ than under any other allocation, because one can simply subtract the constant $\max _{y} S_{j}(y)$ on both sides. Truthful bidding is always optimal and, therefore, $U_{i} \in \mathcal{S}_{i}$.

Second, bidder $i$ must be indifferent between all $S_{i} \in \mathcal{S}_{i}$ and $U_{i}$. For all $S_{i} \in \mathcal{S}_{i}$, it holds that for all other bidding functions $T_{i}$ of bidder $i$ and all bidding functions $S_{j} \in \mathcal{S}_{j}$, the surplus of $S_{i}$ is at least as large as for $T_{i}$ or strictly higher than for $T_{i}$ for at least one $S_{j}$. Recall that the surplus from $U_{i}$ is at least as large as from $S_{i}$. As a result, the strategy $S_{i}$ is iteratively not dominated if and only if for all $S_{j} \in \mathcal{S}_{j}$, the surplus of $S_{i}$ and $U_{i}$ is the same for all $S_{j} \in \mathcal{S}_{j}$. 
We now prove that any profile $S \in \mathcal{S}$ strictly implements the efficient allocation, i.e., $\sum S_{i}\left(x_{i}^{*}\right)>\sum S_{i}\left(x_{i}\right)$ for all feasible allocations $\mathbf{x} \neq \mathbf{x}^{*}$. First, note that the only share implemented by $\left(U_{i}, S_{j}\right)$ is the efficient share, that is,

$$
U_{i}\left(x_{i}^{*}\right)+S_{j}\left(x_{j}^{*}\right)>U_{i}\left(x_{i}\right)+S_{j}\left(x_{j}\right) \text { for all } \mathbf{x} \neq \mathbf{x}^{*} .
$$

To see this, suppose there is an allocation $\mathbf{y} \neq \mathbf{x}^{*}$ with $U_{i}\left(y_{i}\right)+S_{j}\left(y_{j}\right) \geq U_{i}\left(x_{i}^{*}\right)+S_{j}\left(x_{j}^{*}\right)$. As bidder $j$ is indifferent between $U_{j}$ and $S_{j}$, we have that $U_{j}\left(y_{j}\right)+U_{i}\left(y_{i}\right)=U_{j}\left(x_{j}^{*}\right)+U_{i}\left(x_{i}^{*}\right)$. Strict concavity of $U$ implies that there is a unique efficient allocation, implying that $\mathbf{y}=$ $\mathbf{x}^{*}$, a contradiction. Hence, $\left(U_{i}, S_{j}\right)$ implements only the efficient share. The next step uses this property to show that $\left(S_{i}, S_{j}\right)$ also implements the efficient allocation. Again by contradiction, let $\hat{\mathbf{x}} \neq \mathbf{x}^{*}$ be the allocation implemented by $\left(S_{i}, S_{j}\right)$. Bidder $i$ is indifferent between $S_{i}$ and $U_{i}$, so $U_{i}\left(\hat{x}_{i}\right)+S_{j}\left(\hat{x}_{j}\right)=U_{i}\left(x_{i}^{*}\right)+S_{j}\left(x_{j}^{*}\right)$, contradicting inequality (6). As a result, $\left(S_{i}, S_{j}\right)$ must implement the efficient allocation.

The proof that the VCG prices depend on the process of IEDS is constructive. We construct a sequence of eliminations that ends with a set of undominated strategies. Strategies in the set will have the desired properties. To show that a strategy is dominated, one needs to find an alternative strategy that yields weakly higher utility against all admissible strategy profiles of the other bidders and a strictly higher utility against some admissible strategy profiles. Above, we have seen that bidding $U_{i}$ is always optimal. In the subsequent three steps of iterative elimination, we have to find only a strategy $S_{j}$ to show that the alternative of truthful bidding is strictly preferred.

Let $\mathcal{B}$ be the set of all bidding functions, i.e., the set of all $S:[\underline{\theta}, \bar{\theta}] \times[0,1] \rightarrow \mathbb{R}_{+}$. Note that the optimality of a function depends on the type $\theta_{i}$.

Step 1 . Strategies $S_{i}$ for which there exists $\tilde{x}<1$ such that $S_{i}(\tilde{x})>U_{i}(\tilde{x})$ are dominated. Bidder $j$ uses the bidding function

$$
S_{j}(x)= \begin{cases}\max _{y} S_{i}(y)+S_{i}(\tilde{x}) & \text { for } x=1 \\ \max _{y} S_{i}(y) & \text { for } x=1-\tilde{x} \\ 0 & \text { else. }\end{cases}
$$

The bidding profile $S$ implements the allocation in which bidder $i$ wins $\tilde{x}$ and bidder $j$ wins $1-\tilde{x}$, since ties are broken in favor of interior allocations. Bidder $i$ 's surplus is $U_{i}(\tilde{x})-S_{i}(\tilde{x})<0$, whereas the surplus from bidding truthfully is nonnegative. Remove these dominated strategies to obtain $\mathcal{S}^{1} \subset \mathcal{B}$.

Step 2. Strategies are dominated that satisfy $S_{i}(1)>V_{i}(\underline{\theta})$. Note that for low types, $V_{i}(\underline{\theta})>U_{i}(1)$. For high types it can be the case that $\bar{x}_{i}=1$, so $U_{i}(1)=V_{i}(\underline{\theta})$. Bidder $j$ bids $S_{j}(x)=0$ for $x<1$ and $S_{j}(1)=S_{i}(1)-\varepsilon$, with $\varepsilon \in\left(0, S_{i}(1)-V_{i}(\underline{\theta})\right)$. Bidder $i$ wins the full supply at a price higher than utility. Truthful bidding is, therefore, strictly better against this strategy profile of other bidders. Remove these dominated strategies to get $\mathcal{S}^{2} \subset \mathcal{S}^{1}$.

Step 3. Strategies are dominated for which there exists $\tilde{x} \in\left[\underline{x}_{i}, \bar{x}_{i}\right]$ with $U_{i}\left(\tilde{x}_{i}\right)>S_{i}(\tilde{x})$. Let $x^{\prime} \in \arg \max _{y} S_{i}(y)$. Let $\varepsilon \in\left(0, U_{i}(\tilde{x})-S_{i}(\tilde{x})\right)$. In the case of $\tilde{x}<1$, suppose bidder $j$ uses the bidding function $S_{j}(1)=S_{i}\left(x^{\prime}\right)+\varepsilon, S_{j}(1-\tilde{x})=S_{i}\left(x^{\prime}\right)-S_{i}(\tilde{x})$, and $S_{i}(x)=0$ for all other $x$. Under this bidding function, bidder $i$ wins nothing and gets zero surplus. If 
the bid on $\tilde{x}$ is raised to $U_{i}(\tilde{x})$, then bidder $i$ wins $\tilde{x}$ and gets strictly positive surplus. For $\tilde{x}=1$, let $S_{j}(1)=S_{i}(1)+\varepsilon$ and 0 otherwise. Bidder $i$ wins nothing if the bid is below true utility level and wins the full supply if the bid equals utility. The set $\mathcal{S} \subset S^{2}$ is obtained by eliminating these dominated strategies.

After the three steps of elimination, all remaining strategies implement the efficient allocation. To see this, let bidders use the admissible strategy profile $S \in \mathcal{S}$. The value jointly expressed for the efficient allocation is higher than the value jointly expressed for any other feasible allocation $\mathbf{x}$. Let $x_{i}<1$ for all $i$. Then

$$
S_{i}\left(x_{i}\right)+S_{j}\left(x_{j}\right) \leq U_{i}\left(x_{i}\right)+U_{j}\left(x_{j}\right) \leq U_{i}\left(x_{i}^{*}\right)+U_{j}\left(x_{j}^{*}\right)=S_{i}\left(x_{i}^{*}\right)+S_{j}\left(x_{j}^{*}\right),
$$

where the first inequality follows from Step 1, the second inequality follows from the definition of efficiency, and the last equality follows from Steps 1, 2, and 3. For an allocation such that there is an $i$ with $x_{i}=1$, we have

$$
S_{i}\left(x_{i}\right)+S_{j}(x)=S_{i}(1) \leq V_{i}(\underline{\theta}) \leq U_{i}\left(x_{i}^{*}\right)+U_{j}\left(x_{j}^{*}\right)=S_{i}\left(x_{i}^{*}\right)+S_{j}\left(x_{j}^{*}\right),
$$

where the first equality follows from Step 1, the first inequality follows from Step 2, the second inequality follows from the definition of efficiency, and the last equality ffollows rom Steps 1, 2, and 3. All strategy profiles in $\mathcal{S}$ implement the efficient allocation. There are no further dominated strategies, as any strategy that survives IEDS yields the same expected utility as bidding truthfully.

To see that the VCG prices depend on the chosen strategy profile, consider a bidder with $\theta_{i}$ sufficiently small so that $V_{i}(\underline{\theta})<U_{i}(1)$ and the other player has the lowest possible type $\underline{\theta}$. The value of the efficient allocation is $V\left(\theta_{i}, \underline{\theta}\right)$. Suppose bidder $i$ chooses $S_{i} \in \mathcal{S}_{i}$ with $S_{i}(x)=U_{i}(x)$ for $x<1$ and $S_{i}(1)=V_{i}(\underline{\theta})$, and the other bidder plays $S_{j}=U_{j}$. Hence, the VCG price for bidder $j$ is $V_{i}(\underline{\theta})-U_{i}\left(x_{i}^{*}\right)$. If the strategy profile was such that $S_{i}=U_{i}$, then the VCG price would be strictly less than that and equal to $U_{i}(1)-U_{i}\left(x_{i}^{*}\right)$. Note that the strict inequality and continuity imply that the difference in VCG prices holds for an open set of types. Similarly, if Step 1 was such that we also eliminate $S_{i}(1)>U_{i}(1)$, then the first VCG price would not be possible.

\section{REFERENCES}

Ausubel, Lawrence M. (2004), "An efficient ascending-bid auction for multiple objects.” American Economic Review, 94, 1452-1475. [1274]

Ausubel, Lawrence M. and Oleg V. Baranov (2014), "Market design and the evolution of the combinatorial clock auction." American Economic Review: Papers \& Proceedings, 104, 446-451. [1276, 1297]

Ausubel, Lawrence M. and Oleg V. Baranov (2019), "Core-selecting auctions with incomplete information." Unpublished paper, Forthcoming International Journal of Game Theory. [1278]

Ausubel, Lawrence M., Peter Cramton, and Paul Milgrom (2006), “The clock-proxy auction: A practical combinatorial auction design.” In Combinatorial Auctions (P. Cramton, Y. Shoham, and R. Steinberg, eds.). MIT Press. [1272, 1276, 1284] 
Bichler, Martin, Pasha Shabalin, and Jürgen Wolf (2013), "Do core-selecting combinatorial clock auctions always lead to high efficiency? An experimental analysis of spectrum auction designs." Experimental Economics, 16, 511-545. [1276]

BT (2015), "Response to: Public sector spectrum release (PSSR) award of the $2.3 \mathrm{GHz}$ and 3.4 GHz bands." http://goo.gl/xaoDKY, accessed on January 8, 2019. [1275]

Cramton, Peter (2013), "Spectrum auction design." Review of Industrial Organization, 42, 161-190. [1272, 1276, 1277]

Cramton, Peter and Axel Ockenfels (2017), “The German 4G spectrum auction: Design and behaviour." Economic Journal, 127, F305-F324. [1274]

Day, Robert and Peter Cramton (2012), "Quadratic core-selecting payment rules for combinatorial auctions." Operations Research, 60, 588-603. [1278]

Day, Robert and Paul Milgrom (2008), “Core-selecting package auctions.” International Journal of Game Theory, 36, 393-407. [1278]

Erdil, Aytek and Paul Klemperer (2010), "A new payment rule for core-selecting package auctions." Journal of the European Economic Association, 8, 537-547. [1278]

Goeree, Jacob K. and Yuanchuan Lien (2016), "On the impossibility of core-selecting auctions." Theoretical Economics, 11, 41-52. [1278]

Gretschko, Vitali, Stephan Knapek, and Achim Wambach (2017), "Bidding complexities in combinatorial clock auctions." In Handbook of Spectrum Auction Design (Martin Bichler and Jacob Goeree, eds.). Cambridge University Press. [1276]

Grimm, Veronika, Frank Riedel, and Elmar Wolfstetter (2003), "Low price equilibrium in multi-unit auctions: The GSM spectrum auction in Germany." International Journal of Industrial Organization, 21, 1557-1569. [1271]

Janssen, Maarten and Vladimir A. Karamychev (2016), "Spiteful bidding and gaming in combinatorial clock auctions." Games and Economic Behavior, 100, 186-207. [1276]

Kroemer, Christian, Martin Bichler, and Andor Goetzendorf (2016), “(Un)expected bidder behavior in spectrum auctions: About inconsistent bidding and its impact on efficiency in the combinatorial clock auction." Group Decision and Negotiation, 25, 31-63. [1291]

Laffont, Jean-Jacques and Jean Tirole (1988), “The dynamics of incentive contracts." Econometrica, 56, 1153-1175. [1273, 1284]

Levin, Jonathan and Andrzej Skrzypacz (2016), "Properties of the combinatorial clock auction.” American Economic Review, 106, 2528-2551. [1272, 1275, 1276, 1278, 1279, $1281,1285,1291]$

Milgrom, Paul (2004), Putting Auction Theory to Work. Cambridge University Press. [1274]

Ofcom (2012), "Assessment of future mobile competition and award of $800 \mathrm{MHz}$ and 2.6 GHz." http://goo.gl/KfQBsX, accessed on January 8, 2019. [1275] 
Ofcom (2014), "Public sector spectrum release (PSSR) award of the $2.3 \mathrm{GHz}$ and $3.4 \mathrm{GHz}$ bands.” http://goo.gl/L4FjnM, accessed on January 8, 2019. [1275, 1278]

Ofcom (2015), “Annex 8-Recent European awards.” http://goo.gl/CYwDbM. [1291]

Power Auctions LLC (2015), "Auction design considerations for the public sector spectrum release, prepared for Hutchison 3G UK." http://goo.gl/rwacrm, accessed on January 8 , 2019. [1278]

Telekom Austria (2013), "Results of the Austrian spectrum auction." http://goo.gl/ ZZV8eB, accessed on January 8, 2019. [1297]

Co-editor Simon Board handled this manuscript.

Manuscript received 12 February, 2018; final version accepted 2 May, 2019; available online 14 May, 2019. 